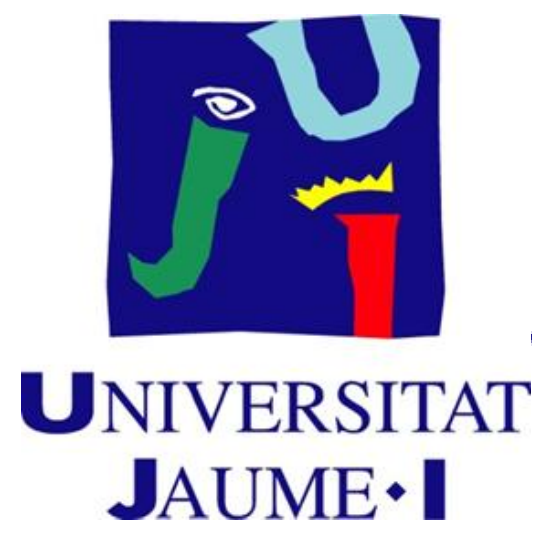

\title{
Calidad de vida en pacientes con
}

\section{ERCA en tratamiento con}

\section{hemodiálisis vs diálisis peritoneal. Revisión integradora.}

Memoria presentada para optar al título de Graduado o Graduada en Enfermería de la Universitat Jaume I presentada por

\author{
María Oriola Alba
}

Curso académico 2016-2017.

Este trabajo ha sido realizado bajo la tutela de Eladio Joaquín Collado Boira. 



\section{Solicitud del alumno/a para el depósito y defensa del TFG}

Yo, MariaOriola Alba, con NIF 53727867M alumna de cuarto curso del Grado en

Enfermería de la Universitat Jaume I, expongo que durante el curso académico 2016/2017.

- He superado al menos 168 créditos ECTS de la titulación

- Cuento con la evaluación favorable del proceso de elaboración de mi TFG.

Por estos motivos, solicito poder depositar y defender mi TFG titulado "Calidad de vida en pacientes con ERCA en tratamiento con hemodiálisis vs diálisis peritoneal. Revisión integradora.”, tutelado por el profesor Eladio J. Collado Boira defendido en lengua española, en el período de 31 de mayo, 2017.

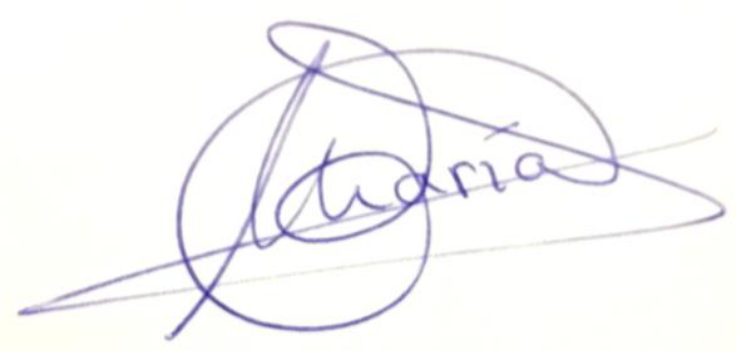

Firmado: MariaOriola Alba

Castellón de la Plana, 16 de mayo de 2017 


\section{Agradecimientos.}

Quiero mostrar mi agradecimiento a todas aquellas personas que me han apoyado en la realización de este trabajo.

A mi tutor del trabajo de final de grado, Eladio Joaquín Collado Boira, por su confianza, su disposición y los consejos y ayuda recibida en cada una de las tutorías.

A mi familia por el apoyo proporcionado durante los cuatro años de grado, y sobre todo en la recta final.

A mi pareja, Josep Ortiz, por aguantarme y por la paciencia y los ánimos que siempre me ha prestado en los momentos de agobio.

A mis compañeros de promoción, por todos los momentos vividos durante la carrera.

A la Universitat Jaume I de Castellón.

Gracias de todo corazón. 


\section{GLOSARIO DE ACRÓNIMOS}

- ERCA: Enfermedad Renal Crónica Avanzada.

- QOL: Calidad de Vida.

- HD: Hemodiálisis.

- PD: Diálisis Peritoneal.

- MeSH: Medical SubjectsHeading.

- DeCS: Descriptores en Ciencias de la Salud.

- OMS: Organización Mundial de la Salud.

- CASPe: Herramienta de calidad metodológica.

- STROBE: Herramienta de calidad metodológica en estudios observacionales.

- KDQOL-SF: Kidney Disease Quality of Life Short Form.

- WHOQOLBREF: World Health Organization Quality of Life Instrument. 


\section{ÍNDICE}

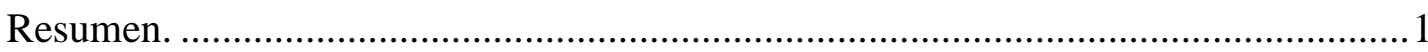

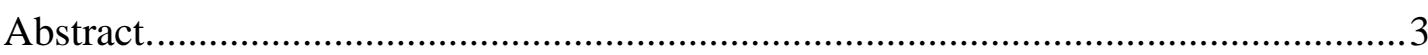

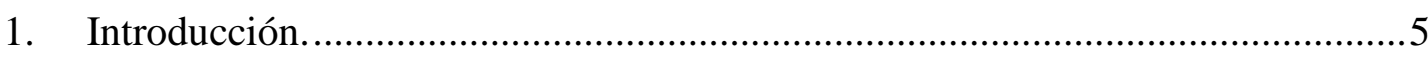

1.1. Enfermedad Renal Crónica (ERC). ..........................................................

1.1.1. Clasificación ...................................................................................

1.1.2. Epidemiología..................................................................................

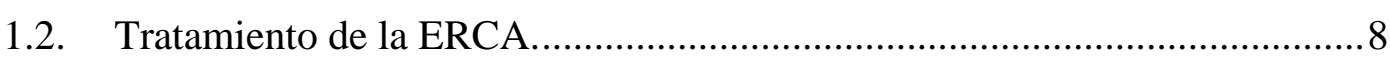

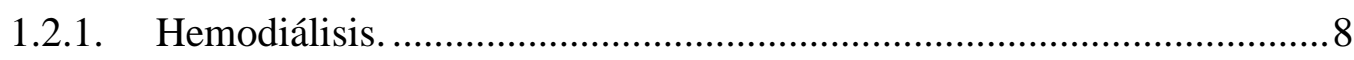

1.2.2. Diálisis peritoneal. ..........................................................................

1.3. Calidad de Vida relacionada con Salud. ……………….............................

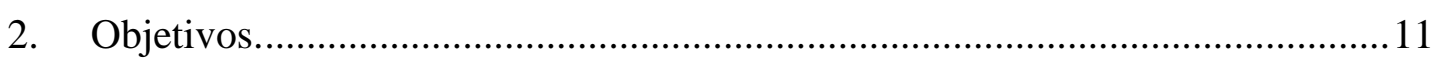

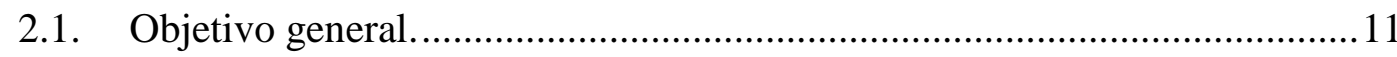

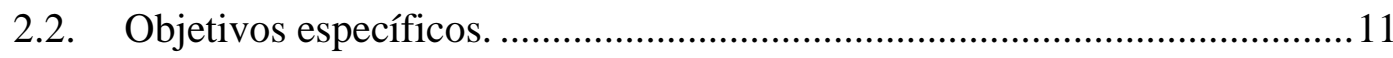

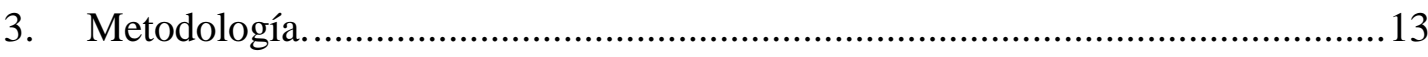

3.1. Identificación del problema o pregunta. ……………………………............13

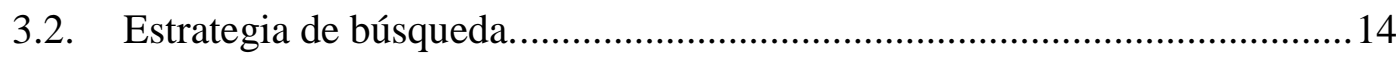

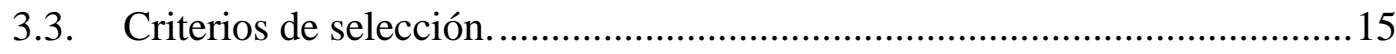

3.3.1. Criterios de inclusión..........................................................................15

3.3.2. Criterios de exclusión. .........................................................................15

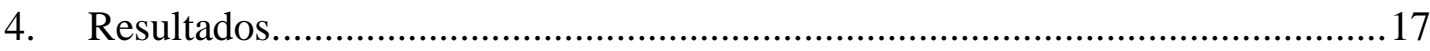

4.1. Resultados de la búsqueda...................................................................... 
4.2. Estudios excluidos.

4.3. Características de los estudios incluidos...............................................22

4.4. Evaluación de la calidad metodológica......................................................22

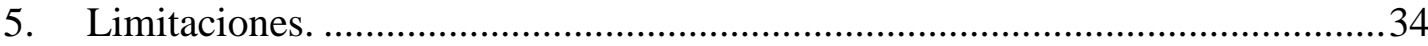

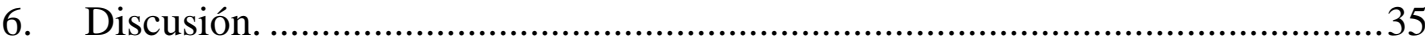

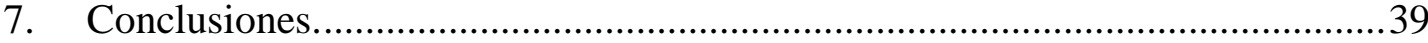

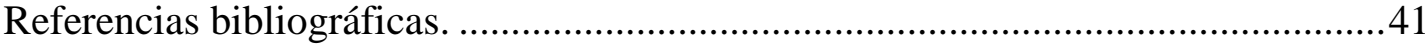

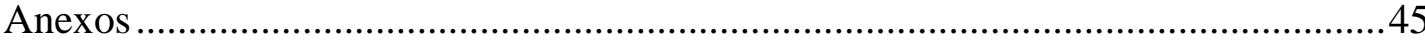

Anexo 1. Herramienta de calidad metodológica CASPe.........................................45

Anexo 2. Herramienta de calidad metodológica STROBE. ....................................49 


\section{ÍNDICE DE TABLAS}

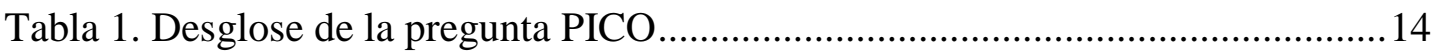

Tabla 2. Lenguaje natural y descriptores DeSC y MeSH..........................................14

Tabla 3. Relación de artículos recuperados en total en el conjunto de las bases de

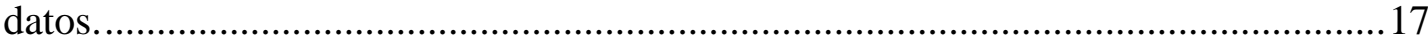

Tabla 4. Detalles de la búsqueda bibliográfica en la base de datos CINAHL............18

Tabla 5. Detalles de búsqueda bibliográfica en la base de datos PubMed. .................19

Tabla 6. Detalles de la búsqueda bibliográfica en la base de datos ProQuest............19

Tabla 7. Detalles de la búsqueda bibliográfica en la base de datos Scopus. ...............20

Tabla 8. Características de los estudios seleccionados...........................................23

Tabla 9. Principales conclusiones de los estudios................................................25

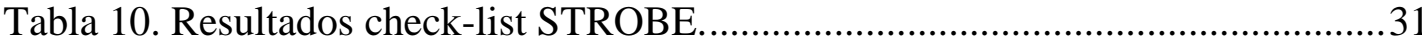

Tabla 11. Resultados herramienta lectura crítica CASPe.........................................32 


\section{ÍNDICE GRÁFICOS}

Gráfica 1. Etapas de la Enfermedad Renal Crónica. ....................................................

Gráfica 2. Relación en porcentaje de la selección y descarte de los estudios

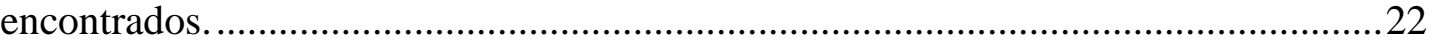

Gráfica 3. Relación en porcentaje de los estudios seleccionados.............................22

Gráfica 4. Porcentaje de Calidad Medtodológica........................................................30 


\section{ÍNDICE ILUSTRACIONES}

Ilustración 1. Diagrama de flujo de la revisión.

.21 
Calidad de vida en pacientes con ERCA en tratamiento con hemodiálisis vs diálisis peritoneal. Revisión integradora.

\section{Resumen.}

Introducción: La enfermedad renal crónica avanzada (ERCA) es una patología con elevada prevalencia e incidencia a nivel mundial, suponiendo un grave problema de salud pública que va en aumento. En la última etapa de la ERCA, las funciones del riñón deben ser reemplazadas. Las técnicas de sustitución renal tienen un fuerte impacto sobre el estilo de vida de los pacientes, y por tanto un cambio en su calidad de vida (QOL).

\section{Objetivos:}

- Objetivo general: comparar la calidad de vida de los pacientes con ERCA tratados con hemodiálisis Vs los tratados con diálisis peritoneal.

\section{- Objetivo específico:}

- Ayudar a los profesionales sanitarios a conocer las últimas evidencias científicas en relación a la calidad de vida que proporcionan los distintos métodos de diálisis, para compartir la información y poder realizar recomendaciones a los pacientes de ERCA y seleccionar el método de diálisis más adecuado.

- Determinar las principales variables clínicas y sociodemográficas relacionadas con la calidad de vida en los pacientes sometidos a HD y DP.

Metodología: Revisión integradora de la bibliografía. Bases de datos: CINAHL, PubMed, Scopus y ProQuest. Criterios de inclusión: revisiones, estudios cuantitativos, descriptivos observacionales y ensayos clínicos. Últimos 5 años. Herramienta de calidad metodológica CASPe y Strobe.

Resultados:Se obtuvieron 14 estudios con los que realizar la revisión integradora.

De los cuales hay 2 revisiones sistemática y 12 estudios observacionales.

Conclusiones: No se encuentran diferencias estadísticamente significativas en la QOL en términos de salud en cuanto a un tipo de tratamiento u otro.

Palabras clave: hemodiálisis, diálisis, diálisis peritoneal, calidad de vida. 
Calidad de vida en pacientes con ERCA en tratamiento con hemodiálisis vs diálisis peritoneal. Revisión integradora. 
Calidad de vida en pacientes con ERCA en tratamiento con hemodiálisis vs diálisis peritoneal. Revisión integradora.

\section{Abstract.}

Introduction: Advanced chronic kidney disease (ACKD) is a pathology with high prevalence and presence worldwide, posing an increasing serious public health issue. In the last stage of the ACKD kidney functions should be replaced. Renal replacement techniques have a strong impact on the patients' lifestyle and thus imply a change in their quality of life (QOL).

\section{Objectives:}

- Overall objective: Comparing the patients' quality of life with ACKD treated with hemodialysis vs. those treated with peritoneal dialysis.

\section{- Specific objective:}

- Helping health professionals to know the latest scientific evidence regarding the quality of life offered by different diagnostic methods in order to share information and make recommendations to ACKD patients while choosing the best dialysis method for them.

- Conclude the main clinical and socio-demographical variables associated to the quality of life in patients treated to HD and PD.

Methodology:Unifying review of the bibliography. Databases: CINAHL, PubMed, Scopus and ProQuest. Inclusion criteria: reviews, quantitative studies, observational descriptions and clinical trials. Last 5 years. Tool of methodological quality CASPe and Strobe.

Results:14 studies were obtainedto perform the unifying review. From them,2 systematic reviews and 12observational studies were made.

Conclusions: There are no significant differences in QOL in terms of health while talking about one type of treatment or another.

Key words:hemodialysis, haemodialysis, dialysis, peritoneal dialysis, quality of life, qol. 
Calidad de vida en pacientes con ERCA en tratamiento con hemodiálisis vs diálisis peritoneal. Revisión integradora. 
Calidad de vida en pacientes con ERCA en tratamiento con hemodiálisis vs diálisis peritoneal. Revisión integradora.

\section{Introducción.}

\subsection{Enfermedad Renal Crónica (ERC).}

La función que desarrolla el riñón en el organismo, consiste en filtrar los desechos del metabolismo celular y el exceso de líquidos que transporta la sangre, los cuales posteriormente son excretados en la orina. La pérdida gradual de la función renal es lo que se denomina enfermedad renal crónica o insuficiencia renal crónica. ${ }^{(1)}$

La enfermedad renal crónica (ERC) se define como la alteración de la estructura renal o de su función, evidenciado por indicadores biológicos de daño renal en orinaysangre o a través de pruebas de imagen, durante un periodo igual o mayor a 3 meses, o por un filtrado glomerular teórico (FGt) menor a $60 \mathrm{ml} / \mathrm{min} / 1,73 \mathrm{~m}^{2}$, independientemente de la etiología del proceso.. ${ }^{(2)(3)(4)}$

\subsubsection{Clasificación.}

La enfermedad renal crónica se divide en 5 etapas. Cada etapa se determina según el porcentaje de función renal que mantenga. (Gráfica 1)

- Enfermedad renal en etapa temprana: En esta etapa muchas personas no presentan signos o síntomas de daño renal, lo que hace que sea muy difícil su diagnóstico y tratamiento. ${ }^{(5)}$

Etapa 1: Daño renal con FGTnormal o aumentada (> $\left.90 \mathrm{ml} / \mathrm{min} / 1,73 \mathrm{~m}^{2}\right)$. Esta etapa se caracteriza por que el paciente presenta presión arterial alta, infecciones del tracto urinario y análisis de orina anormal. ${ }^{(5)}$

Etapa 2: Daño renal con disminución leve de FGT (entre 60 y 89 $\mathrm{ml} / \mathrm{min} / 1,73 \mathrm{~m}^{2}$ ). Presenta presión arterial alta, infecciones del tracto urinario, análisis de orina anormal. ${ }^{(5)}$

Etapa 3: Disminución moderada en FGT (entre 30 y $59 \mathrm{ml} / \mathrm{min} / 1,73 \mathrm{~m}^{2}$ ). Presenta desnutrición, dolor óseo, neuropatías periféricas caracterizadas por la 
Calidad de vida en pacientes con ERCA en tratamiento con hemodiálisis vs diálisis peritoneal. Revisión integradora.

presencia de hormigueo y entumecimiento, disminución de la función mental y de la sensación de bienestar. ${ }^{(5)}$

\section{- Enfermedad renal en etapa tardía:}

Etapa 4: Disminución severa del TFG, con cifras entre15 y 29ml/min $/ 1,73 \mathrm{~m}^{2}$. Presencia de complicaciones graves tales como anemia, presión arterial alta y enfermedades óseas. ${ }^{(5)}$

- Enfermedad renal en etapa avanzada o terminal:es la última etapa en la historia natural de la ERC. Es el resultado del deterioro crónico, progresivo, pluripatológico e irreversible de la función renal. Ambos riñones han dejado de desarrollar su función de filtración, secreción y endocrina o prácticamente estas son nulas, yen cuya situación, si no se realizara un tratamiento sustitutivo a través de técnicas de diálisis o un trasplante renal, llevarían a la muerte. ${ }^{(4)(5)}$

Etapa 5: Insuficiencia renal. FGT inferior a $15 \mathrm{ml} / \mathrm{min} / 1,73 \mathrm{~m}^{2}$. Uremia y azoemia (acumulación de productos nitrogenados de desecho en la sangre), anemia, desnutrición, hiperparatiroidismo, presión arterial alta, edemas por incremento de la presión oncótica(manos, piernas, ojos, espalda baja), y disnea. ${ }^{(5)}$ 
Calidad de vida en pacientes con ERCA en tratamiento con hemodiálisis vs diálisis peritoneal. Revisión integradora.

\section{La Fundación Nacional del Riñón (National Kidney Foundation) ha dividido la enfermedad renal crónica en 5 etapas. Cada etapa se determina en función del porcentaje de la función renal restante.}

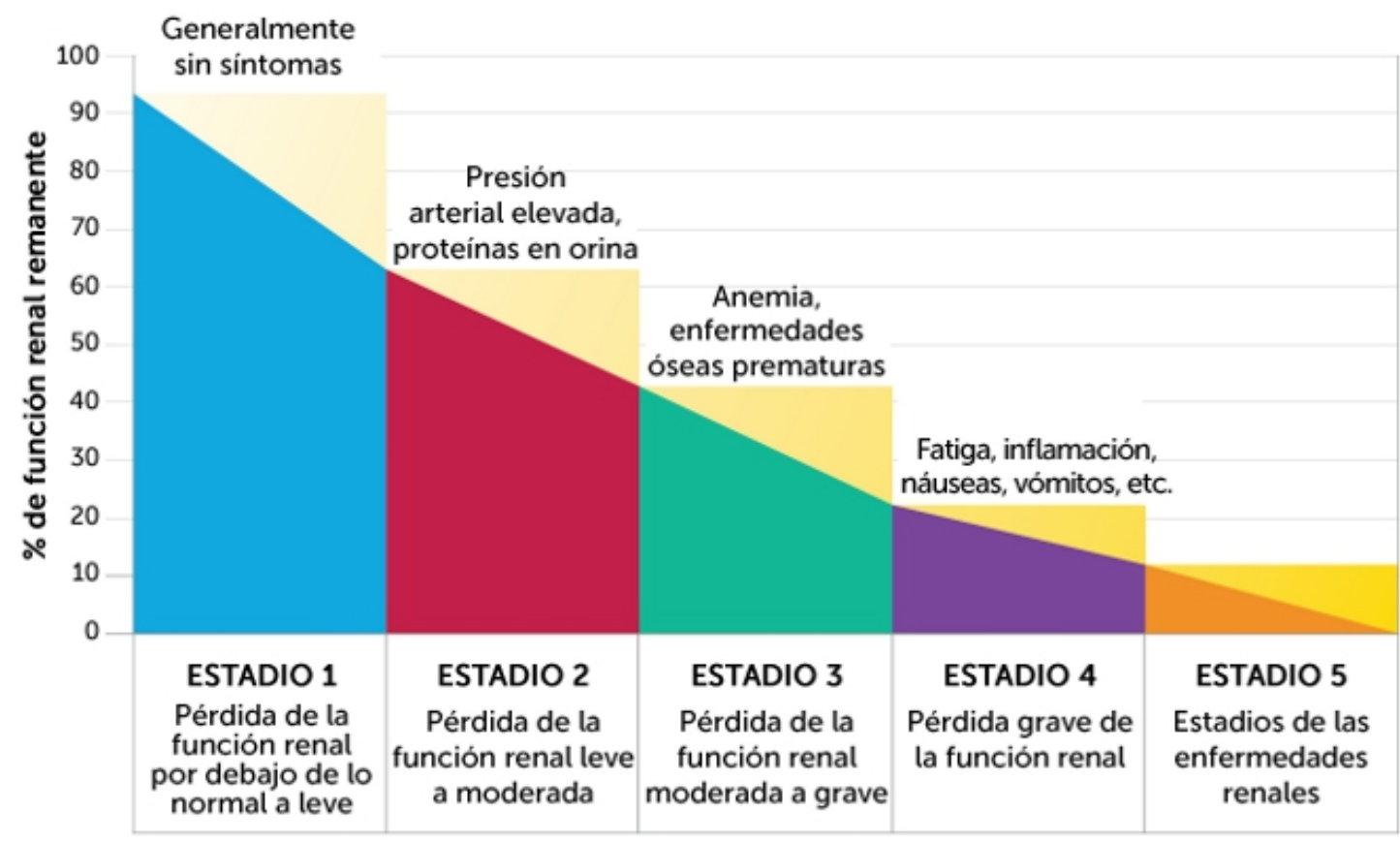

Stages of CKD

Adaptado de las directrices de la Iniciativa sobre la calidad de los desenlaces de la enfermedad renal (Kidney Disease Outcomes Quality Initiative - KDOQI), de la Fundación Nacional del Riñón (National Kidney Foundation)

Gráfica 1. Etapas de la Enfermedad Renal Crónica.

\subsubsection{Epidemiología.}

La enfermedad renal crónica (ERC) se ha convertido en un importante problema de salud pública, que ha adquirido proporciones epidémicas ${ }^{(3)(4)(6)}$. Afecta a un porcentaje significativo de la población, hecho justificable debido a que los principales factores etiológicos sontrastornos de alta prevalencia como el envejecimiento, la hipertensión arterial (HTA), la diabetes Mellitus (DM) y la enfermedad vascular. Sin embargo, la verdadera prevalencia de la enfermedad es difícil de evaluar, ya que los estados iníciales usualmente son asintomáticos, dada la gran capacidad de adaptación celular de las nefronas al daño celular. ${ }^{(4)}$

Se estima en población general adulta a nivel mundial, que aproximadamente entre un 8 y un $10 \%$ tiene algún daño en el riñón, evidenciado por proteinuria o microalbúminuria y que en caso de incluirsela hematuria se incrementa hasta un $18 \%$. ${ }^{(7)}$ 
Calidad de vida en pacientes con ERCA en tratamiento con hemodiálisis vs diálisis peritoneal. Revisión integradora.

Según los resultados del estudioepidemiológico sobre la insuficiencia renal crónica en España (EPIRCE), diseñado para obtener datos sobre la prevalencia de la ERC en España y promovido por la Sociedad Española de Nefrología (SEN), con el apoyo del Ministerio de Sanidad yConsumo, el 9,24\% de la población adulta sufre algúngrado de ERC1. El 6,83\% de la población presenta unadisminución del filtrado glomerular (FG) por debajo de60 ml/min/1,73 $\mathrm{m}^{2}$, siendo este porcentaje del $20,6 \%$ en mayores de 64 años. $^{(3)}$

En la Comunidad Valenciana la tasa provisional de prevalencia de enfermos renales (residentes y no residentes) en 2015 era de 6.527 casos.En relación a la distribución del último tratamiento de sustitución renal prescrito, la tasa de prevalencia en 2015 era de 3.256 personas en tratamiento con hemodiálisis o hemodiafiltración en hospital/centro, 441 casos en diálisis peritoneal,17 casos en hemodiálisis o hemodiafiltración domiciliaria y 2.809 pacientes trasplantados de riñón. ${ }^{(8)}$

\subsection{Tratamiento de la ERCA.}

Las técnicas de terapia renal sustitutiva son aquellas cuya finalidad es suplir la función del riñón, cuando este presenta un deterioro grande de sus funciones. Existe dos tipos principales de terapia renal sustitutiva, la terapia con hemodiálisis extracorpórea convencional, y la terapia con diálisis peritoneal. Dentro de estas dos principales técnicas se encuentra una variedad de subtipos: hemodiálisis domiciliaria, hemodiálisis autoasistida, diálisis peritoneal automatizada y diálisis peritoneal continua ambulatoria. ${ }^{(9)}$

El uso de estas técnicas, imprescindibles para la supervivencia de estos enfermos, mejora la fisiología general del organismo, y la sensación de bienestar.

La selección de la técnica de depuración renal a utilizar se realizará en función de las características del paciente y de las recomendaciones del nefrólogo ${ }^{(10)}$

\subsubsection{Hemodiálisis.}

La hemodiálisis es una técnica de reemplazo renal extracorpórea, que se utiliza en la insuficiencia renal aguda o en la insuficiencia renal crónica terminal. Se hace circular la 
Calidad de vida en pacientes con ERCA en tratamiento con hemodiálisis vs diálisis peritoneal. Revisión integradora.

sangre a través de un filtro consistente en una membrana semipermeable y que en función de una serie de parámetros de presión osmótica y en presencia de una solución de diálisis actúa como riñón artificial, y realiza su depuración. Las funciones del riñón reemplazadas por esta técnica son las siguientes: excreción de solutos, eliminación del líquido retenido y regulación del equilibrio ácido-base y electrolítico. Esta técnica no suple las funciones endocrinas ni metabólicas del riñón. ${ }^{(10)(11)}$

El tiempo y la frecuencia de las sesiones de tratamiento con hemodiálisis depende de las necesidades de cada paciente. La media de tratamiento es de 4 horas, 3 veces por semana. Puede realizarse en el hospital ( como centro de referencia del paciente con ERCA o en clínicas de diálisis. ${ }^{(10)(11)}$

\subsubsection{Diálisis peritoneal.}

La técnica de diálisis peritoneal, en lugar de utilizar un filtro artificial, hace uso delas características propias del peritoneo como membrana semipermeable, por lo que la sangre no sale del cuerpo. El líquido de diálisis se introduce en el abdomen del paciente a través de un catéter implantado previamente de forma quirúrgica, y se extrae pasado un tiempo, en el que se ha producido el intercambio de solutos en la membrana, realizando así la diálisis. ${ }^{(10)(12)}$ Se realiza con una media de 3 a 5 intercambios al día, dependiendo de las necesidades del paciente, con la ayuda de una cicladora y la mayoría de las veces en régimen nocturno, aprovechando las horas de descanso del paciente. Este tipo de diálisis se realiza en una habitación adaptada en la residencia del paciente, donde es muy importante la higiene y los cuidados de asepsia y antisepsia. ${ }^{(10)}$

\subsection{Calidad de Vida relacionada con Salud.}

Los tratamiento de sustitución de la función renal, generan un cambio en el estilo de vida de los pacientes, y por lo tanto una modificación de la calidad de vida (QOL).

La Organización Mundial de la Salud (OMS) en 1994 definió la calidad de vida como: "la percepción del individuo de su posición en la vida en el contexto de la cultura y el sistema de valores en los que vive y en relación con sus objetivos, expectativas, valores y preocupaciones" ${ }^{(13)(14)}$ 
Calidad de vida en pacientes con ERCA en tratamiento con hemodiálisis vs diálisis peritoneal. Revisión integradora.

La calidad de vida relacionada con la salud es un concepto multidisciplinar, el que se centra en la evaluación subjetiva de la persona y en el cual intervienen factores clínicos como son la enfermedad y la atención sanitaria, y la promoción de la salud sobre su capacidad de mantener un nivel de funcionamiento que le permita llevar a cabo las actividades que anteriormente realizaba y le resultaban satisfactorias. Además, también intervienen factores no clínicos como son la familia, el trabajo, las creencias religiosas, los ingresos y otras circunstancias de la vida. ${ }^{(13)}$

La valoración de la QOL se suele utilizar para obtener información sobre el bienestar del paciente, y es utilizada para el control de la calidad de los cuidados y la atención prestada a los pacientes en tratamiento de diálisis. ${ }^{(14)}$

La QOL es un factor importante a tener en cuenta a la hora de aplicar un tratamiento $u$ otro de sustitución renal. 
Calidad de vida en pacientes con ERCA en tratamiento con hemodiálisis vs diálisis peritoneal. Revisión integradora.

\section{Objetivos.}

\subsection{Objetivo general.}

Realiza una revisión de la literatura para informar sobre el estado actual de la calidad de vida de los pacientes con ERCA tratados con hemodiálisis Vs los tratados con diálisis peritoneal.

\subsection{Objetivos específicos.}

- Ayudar a los profesionales médicos a conocer las discrepancias en cuanto a la calidad de vida que proporcionan los distintos métodos de diálisis, para compartir la información y poder realizar recomendaciones a los pacientes de ERCA y seleccionar el método de diálisis adecuado.

- Determinar las principales variables clínicas y sociodemográficas relacionadas con la calidad de vida en los pacientes sometidos a HD y DP. 
Calidad de vida en pacientes con ERCA en tratamiento con hemodiálisis vs diálisis peritoneal. Revisión integradora. 
Calidad de vida en pacientes con ERCA en tratamiento con hemodiálisis vs diálisis peritoneal. Revisión integradora.

\section{Metodología.}

La metodología empleada para la realización de este estudio se ha basado en una revisión integradora de la literatura con el fin de elaborar un análisis sobre las evidencias científicas acerca de la calidad de vida de pacientes con ERCA sometidos a tratamientos de depuración renal.

Las revisiones de la evidencia científica son estudios secundarios que sintetizan la mejor evidencia científica disponible mediante métodos explícitos rigurosos para identificar, seleccionar, evaluar, analizar y sintetizar los estudios empíricos que permitirán responder a cuestiones específicas ${ }^{(15)}$

En esta revisión se siguieron los siguientes pasos:

- $\quad$ Identificación del problema o pregunta.

- $\quad$ Estrategia de búsqueda bibliográfica

- Establecimiento de los criterios de inclusión y exclusión.

- Limitaciones que hemos tenido al realizar la búsqueda.

- Análisis de la información, interpretación y presentación de esta revisión.

\subsection{Identificación del problema o pregunta.}

El primer paso antes de realizar la búsqueda de información es identificar el problema. Para ello es necesario realizar una pregunta PICO, en la cual se identifique el paciente al que va dirigida la intervención, la intervención en si misma que se pretende realizar, una intervención de comparación y el resultado esperado.

La pregunta PICO que se realizó a la hora de diseñar este estudio fue la siguiente:

¿Las personas con ERCA tratadas con hemodiálisis tiene la misma calidad de vida que las tratadas con diálisis peritoneal ${ }^{\text {Tabla } 1}$. 
Calidad de vida en pacientes con ERCA en tratamiento con hemodiálisis vs diálisis peritoneal. Revisión integradora.

Tabla 1. Desglose de la pregunta PICO

\section{$\mathbf{P}$ (patient)}

I (intervention)

C (comparation)

$\mathbf{O}$ (outcoms)

\begin{tabular}{l} 
Tratadas con hemodiálisis \\
\hline Tratadas con diálisis peritoneal \\
\hline Calidad de Vida
\end{tabular}

\subsection{Estrategia de búsqueda.}

A partir de la pregunta realizada se concretaron las palabras clave que iban a ser utilizadas a la hora de realizar la búsqueda, tanto en lenguaje natural como en lenguaje controlado con el tesauro Descriptores en Ciencias de la Salud (DeCS) y el tesauro Medical SubjectHeadings (MeSH). ${ }^{\text {Tabla } 2}$

Tabla 2. Lenguaje natural y descriptores DeSC y MeSH.

\begin{tabular}{|lll|}
\hline Lenguaje natural & DeCS & MeSH \\
\hline Calidad de Vida & Quality of Life & Quality of Life \\
& QoL & QoL \\
\hline Diálisis peritoneal & Peritoneal dialysis & Peritoneal dialysis \\
\hline $\begin{array}{l}\text { Hemodiálisis } \\
\text { Diálisis extracorporea }\end{array}$ & Renal dialysis & Dialysis \\
\hline
\end{tabular}

Posteriormente se realizó la búsqueda de la literatura científica con los descriptores MeSH "QoL", "Quality of Life", "Peritoneal dialysis", "Haemodilysis" y "Dialysis" en las bases de datos CINAHL, PubMed, Scopus y ProQuest. El rango de fecha de publicación de los artículos se delimitó entre el 2012-2017. En todas las bases de datos consultadas se utilizó el operador booleano OR para buscar un mismo descriptor, y el operador booleano AND para combinar los tres descriptores.

En la base de datos ProQuest la búsqueda avanzada se tuvo que realizar toda en una línea, puesto que sino los resultados encontrados eran numerosos y no se podía acotar correctamente. 
Calidad de vida en pacientes con ERCA en tratamiento con hemodiálisis vs diálisis peritoneal. Revisión integradora.

\subsection{Criterios de selección.}

\subsubsection{Criterios de inclusión.}

- Artículos publicados en los últimos cinco años.

- El idioma de publicación de los artículos sea castellano o inglés.

- Aquellos artículos de los cuales esté disponible el texto completo.

- Estudios que traten la calidad de vida de los pacientes tratados en hemodiálisis y diálisis peritoneal.

- Pacientes mayores de edad.

- Revisiones de la literatura, estudios cuantitativos, ensayos clínicos y estudios descriptivos observacionales.

\subsubsection{Criterios de exclusión.}

- Artículos publicados hace más de cinco años.

- El idioma de publicación no sea ni castellano ni inglés.

- Artículos de los cuales no se disponga del texto completo.

- Artículos que hablen de la terapia de sustitución renal pero no tengan en cuenta la calidad de vida de los pacientes.

- Los pacientes a estudio sean niños.

- Artículos que no sean revisiones de la literatura, estudios cuantitativos, ensayos clínicos o estudios descriptivos observacionales. 
Calidad de vida en pacientes con ERCA en tratamiento con hemodiálisis vs diálisis peritoneal. Revisión integradora. 
Calidad de vida en pacientes con ERCA en tratamiento con hemodiálisis vs diálisis peritoneal. Revisión integradora.

\section{Resultados.}

\subsection{Resultados de la búsqueda.}

Al realizar la búsqueda bibliográfica en las cuatro bases de datos seleccionadas, sin aplicar ningún tipo de limitador, se obtuvieron 1,304 documentos. Aplicando los limitadores correspondientes en cada una de las bases de datos, el número total de artículos recuperados fueron 70 . Tablas 3-7

Tabla 3. Relación de artículos recuperados en total en el conjunto de las bases de datos.

\section{Bases de datos Artículos encontrados sin limitadores \\ Artículos recuperados con la aplicación de limitadores}

\begin{tabular}{|ccc|}
\hline CINAHL & 201 & 6 \\
\hline PubMed & 113 & 11 \\
\hline ProQuest & 847 & 15 \\
\hline Scopus & 143 & 38 \\
\hline & $\mathbf{1 , 3 0 4}$ & $\mathbf{7 0}$ \\
\hline
\end{tabular}


Calidad de vida en pacientes con ERCA en tratamiento con hemodiálisis vs diálisis peritoneal. Revisión integradora.

Tabla 4. Detalles de la búsqueda bibliográfica en la base de datos CINAHL.

\begin{tabular}{|c|c|c|}
\hline $\begin{array}{l}\text { Base de } \\
\text { datos }\end{array}$ & Detalles de la búsqueda & $\begin{array}{l}\text { Artículos } \\
\text { encontrados }\end{array}$ \\
\hline \multirow[t]{7}{*}{ CINAHL } & $\begin{array}{l}\text { (haemodialysis or hemodialysis or dialysis) } \\
\text { AND peritoneal dialysis AND (qol or quiality of } \\
\text { life) }\end{array}$ & 201 \\
\hline & $\begin{array}{l}\text { (haemodialysis or hemodialysis or dialysis) } \\
\text { AND peritoneal dialysis AND (qol or quiality of } \\
\text { life) }\end{array}$ & \multirow[t]{2}{*}{82} \\
\hline & Limitadores: Texto completo. & \\
\hline & $\begin{array}{l}\text { (haemodialysis or hemodialysis or dialysis) } \\
\text { AND peritoneal dialysis AND (qol or quiality of } \\
\text { life) }\end{array}$ & \multirow[t]{2}{*}{39} \\
\hline & $\begin{array}{l}\text { Limitadores: Texto completo; Fecha de } \\
\text { publicación 2012-2017 }\end{array}$ & \\
\hline & $\begin{array}{l}\text { TI (haemodialysis or hemodialysis or dialysis) } \\
\text { AND TI peritoneal dialysis AND (qol or quality } \\
\text { of life) }\end{array}$ & \multirow[t]{2}{*}{6} \\
\hline & $\begin{array}{l}\text { Limitadores: Texto completo; Fecha de } \\
\text { publicación 2012-2017 }\end{array}$ & \\
\hline
\end{tabular}


Calidad de vida en pacientes con ERCA en tratamiento con hemodiálisis vs diálisis peritoneal. Revisión integradora.

Tabla 5. Detalles de búsqueda bibliográfica en la base de datos PubMed.

\begin{tabular}{|c|c|c|}
\hline $\begin{array}{l}\text { Base de } \\
\text { datos }\end{array}$ & Diseño de la búsqueda & $\begin{array}{l}\text { Artículos } \\
\text { encontrados }\end{array}$ \\
\hline \multirow[t]{5}{*}{ PubMed } & $\begin{array}{l}\text { (((haemodialysis) OR dialysis) AND peritoneal } \\
\text { dialysis) AND qol) }\end{array}$ & 113 \\
\hline & $\begin{array}{l}\text { (((haemodialysis) OR dialysis) AND peritoneal } \\
\text { dialysis) AND qol) }\end{array}$ & 38 \\
\hline & Limitadores: Free full text. & \\
\hline & $\begin{array}{l}\text { (((haemodialysis) OR dialysis) AND peritoneal } \\
\text { dialysis) AND qol) }\end{array}$ & 11 \\
\hline & $\begin{array}{l}\text { Limitadores: Free full text; published in the lasst } \\
5 \text { years. }\end{array}$ & \\
\hline
\end{tabular}

Tabla 6. Detalles de la búsqueda bibliográfica en la base de datos ProQuest.

\begin{tabular}{|c|c|c|}
\hline $\begin{array}{l}\text { Base de } \\
\text { datos }\end{array}$ & Diseño de la búsqueda & $\begin{array}{l}\text { Artículos } \\
\text { encontrados }\end{array}$ \\
\hline \multirow[t]{6}{*}{ ProQuest } & $\begin{array}{l}\text { (hemodialysis OR dialysis) AND peritoneal } \\
\text { dialysis AND qol }\end{array}$ & 847 \\
\hline & $\begin{array}{l}\text { ab((hemodialysis OR dialysis) AND peritoneal } \\
\text { dialysis AND qol) }\end{array}$ & 38 \\
\hline & $\begin{array}{l}\text { ab((hemodialysis OR dialysis) AND peritoneal } \\
\text { dialysis AND qol) }\end{array}$ & 19 \\
\hline & Limitadores: Fecha de publicación 2012-2017. & \\
\hline & $\begin{array}{l}\text { ab((hemodialysis OR dialysis) AND peritoneal } \\
\text { dialysis AND qol) }\end{array}$ & 15 \\
\hline & $\begin{array}{l}\text { Limitadores: Fecha de publicación 2012-2017; } \\
\text { Texto completo incluido. }\end{array}$ & \\
\hline
\end{tabular}


Calidad de vida en pacientes con ERCA en tratamiento con hemodiálisis vs diálisis peritoneal. Revisión integradora.

Tabla 7. Detalles de la búsqueda bibliográfica en la base de datos Scopus.

\begin{tabular}{|c|c|c|}
\hline $\begin{array}{l}\text { Base de } \\
\text { datos }\end{array}$ & Diseño de la búsqueda & $\begin{array}{l}\text { Artículos } \\
\text { encontrados }\end{array}$ \\
\hline \multirow[t]{5}{*}{ Scopus } & $\begin{array}{l}\text { Title-abs-key (haemodialysis OR dialysis) AND } \\
\text { Title-abs-key (peritoneal dialysis) AND Title-abs- } \\
\text { key (qol) }\end{array}$ & 143 \\
\hline & $\begin{array}{l}\text { Title-abs-key (haemodialysis OR dialysis) AND } \\
\text { Title-abs-key (peritoneal dialysis) AND Title-abs- } \\
\text { key (qol) }\end{array}$ & 39 \\
\hline & Limitadores: Fecha de publicación 2012-2017. & \\
\hline & $\begin{array}{l}\text { Title-abs-key (haemodialysis OR dialysis) AND } \\
\text { Title-abs-key (peritoneal dialysis) AND Title-abs- } \\
\text { key (qol) }\end{array}$ & 38 \\
\hline & $\begin{array}{l}\text { Limitadores: Fecha de publicación 2012-2017; } \\
\text { Review, Article. }\end{array}$ & \\
\hline
\end{tabular}


Calidad de vida en pacientes con ERCA en tratamiento con hemodiálisis vs diálisis peritoneal. Revisión integradora.

\subsection{Estudios excluidos.}

De los 70 estudios obtenidos en las bases de datos, no todos fueron seleccionados. Por no encontrarse disponible el texto completo de forma abierta $(n=24)$, por encontrarse repetidos $(n=12)$, y por no adecuarse al tema en título y resumen $(n=20)$ se excluyeron un total de 56 estudios, dejando un número final de 14 estudios con los que continuar la revisión y a los que realizar la lectura reflexiva. .lustración 1, Gráfica 2.

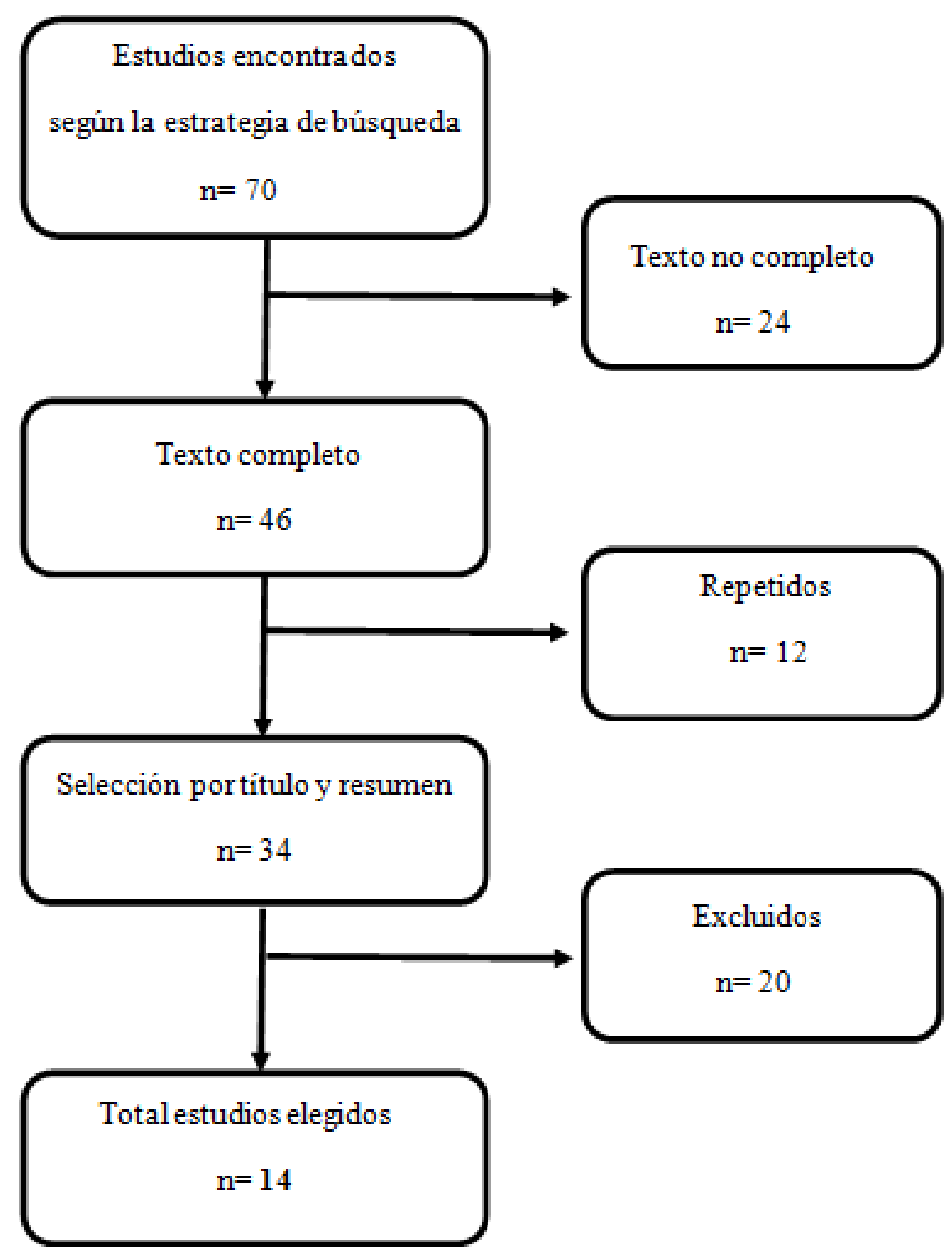

Ilustración 1. Diagrama de flujo de la revisión. 


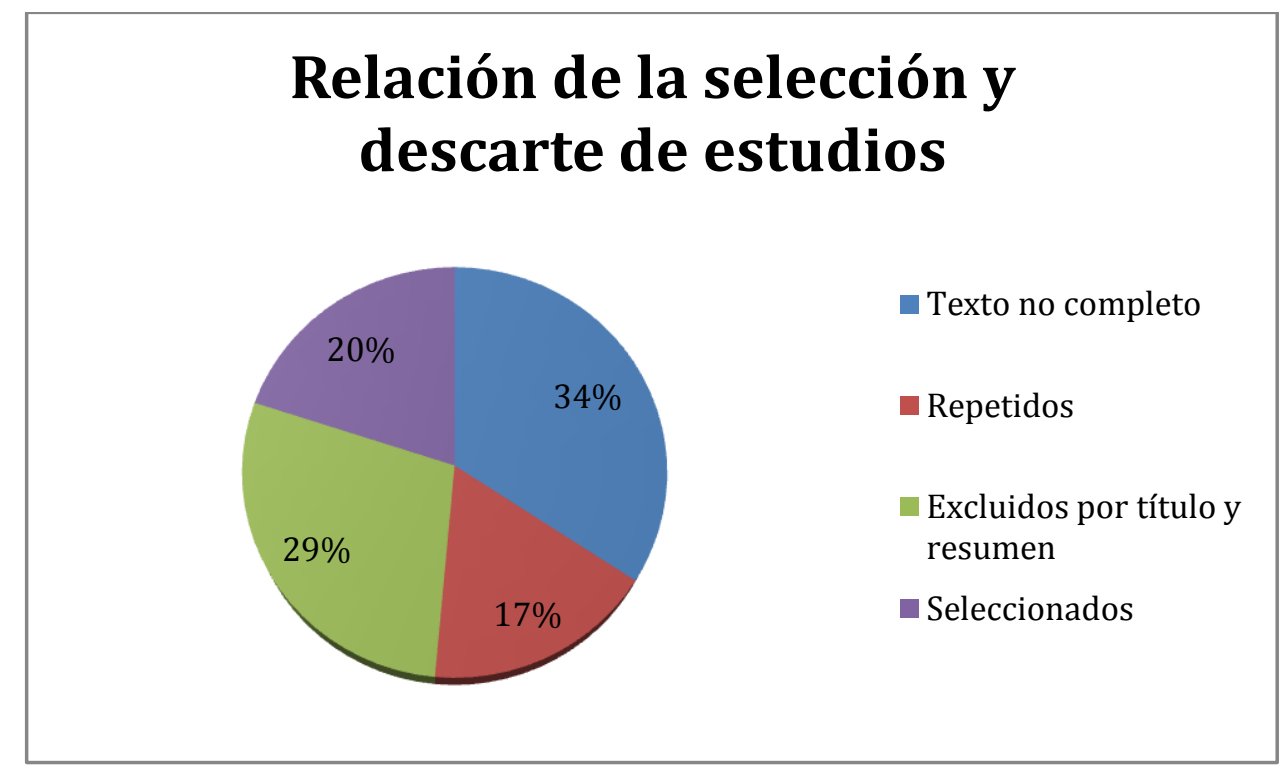

Gráfica 2. Relación en porcentaje de la selección y descarte de los estudios encontrados.

\subsection{Características de los estudios incluidos.}

Doce de los estudios incluidos en esta revisión son estudios de tipo observacional transversal, y los dos restantes son revisiones sistemáticas. Gráfico 3, Tabla ${ }^{8}$ Todos ellos hablan de las terapias de sustitución renal HD y PD, y de su relación con la calidad de vida de los pacientes en tratamiento. ${ }^{\text {Tabla } 9 .}$

\section{Tipos de estudios seleccionados}

घevisiónes sistemáticas $\quad$ Estudios transversales

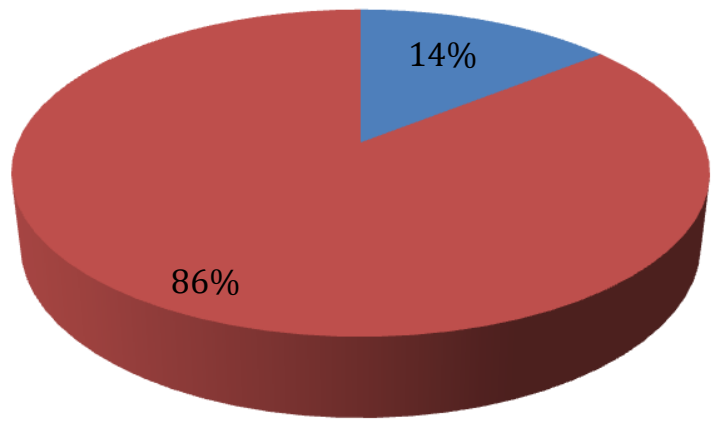

Gráfica 3. Relación en porcentaje de los estudios seleccionados. 
1. Quality of life in chronic haemodialysis and peritoneal Oren, B; Enc, N 2013 Transversal dialysis patients in Turkey and related factors

\begin{abstract}
2. Quality of Life and Self-Efficacy in Three Dialysis Modalities: Incenter Hemodialysis, Home Hemodialysis, and

Home Peritoneal Dialysis

3. Quality of life in hemodialysis and peritoneal dialysis patients in Saudi Arabia
\end{abstract}

\section{Quality of life and mental health in hemodialysis and peritoneal dialysis patients: the role of health beliefs}

Wright, LS; Wilson, L

2015

Transversal

KDQOL-SF

Wakeel, J Al; Harbi, A Al; Bayoumi, M; Al-Suwaida, K; Ghonaim, M Al; Mishkiry, A

$2012 \quad$ Transversal KDQOL-SF

2012

Transversal

STAI 1/STAI 2;

CES-D; MHLC

\begin{tabular}{|c|c|c|c|c|}
\hline $\begin{array}{l}\text { 5. Quality of life and emotional distress between patients on } \\
\text { peritoneal dialysis versus community-based hemodialysis }\end{array}$ & $\begin{array}{l}\text { Griva, K; Kang, A.W.; Yu, Z.L.; } \\
\text { Mooppli, N.K.; Foo, M; Chan, } \\
\text { C.M.; Newman, S.P. }\end{array}$ & 2014 & Transversal & $\begin{array}{l}\text { SF-12 (PCS, MCS) } \\
\text { WHOQOL-BREF }\end{array}$ \\
\hline
\end{tabular}

\begin{tabular}{ll}
\hline 6. Quality of life in end stage renal disease patients & Joshi, V.D. \\
\hline 7. Quality of life in patients on chronic dialysis in South & Tannor, E.K.; Archer, E; \\
Africa: a comparative mixed methods study & Kapembwa, K; Van Schallkwyk,
\end{tabular}

$\begin{array}{ll}\text { Africa: a comparative mixed methods study } & \text { Kapembwa, K; Van } \\ & \text { S.C.; Razeen, D.M. }\end{array}$

$2014 \quad$ Revisión

2017 Transversal KDQOL-SF

2014 $\begin{array}{ll}\text { 8. Quality of life of chronic renal patients in peritoneal } & \text { Carpena Ramos, E.C.; } \\ \text { dialysis and hemodialysis } & \text { Da Silva dos Santos, I; De Vargas } \\ & \text { Zanini, R; Grahl Ramos, J.M }\end{array}$

$\begin{array}{ll}\begin{array}{l}\text { 8. Quality of life of chronic renal patients in peritoneal } \\ \text { dialysis and hemodialysis }\end{array} & \text { Carpena Ramos, E.C.; } \\ & \text { Da Silva dos Santos, I; De Vargas } \\ & \text { Zanini, R; Grahl Ramos, J.M. }\end{array}$

9. Dyadic Relationship and Quality of Life Patients with Barata, N.R.E.R.C.
2015

Transversal 


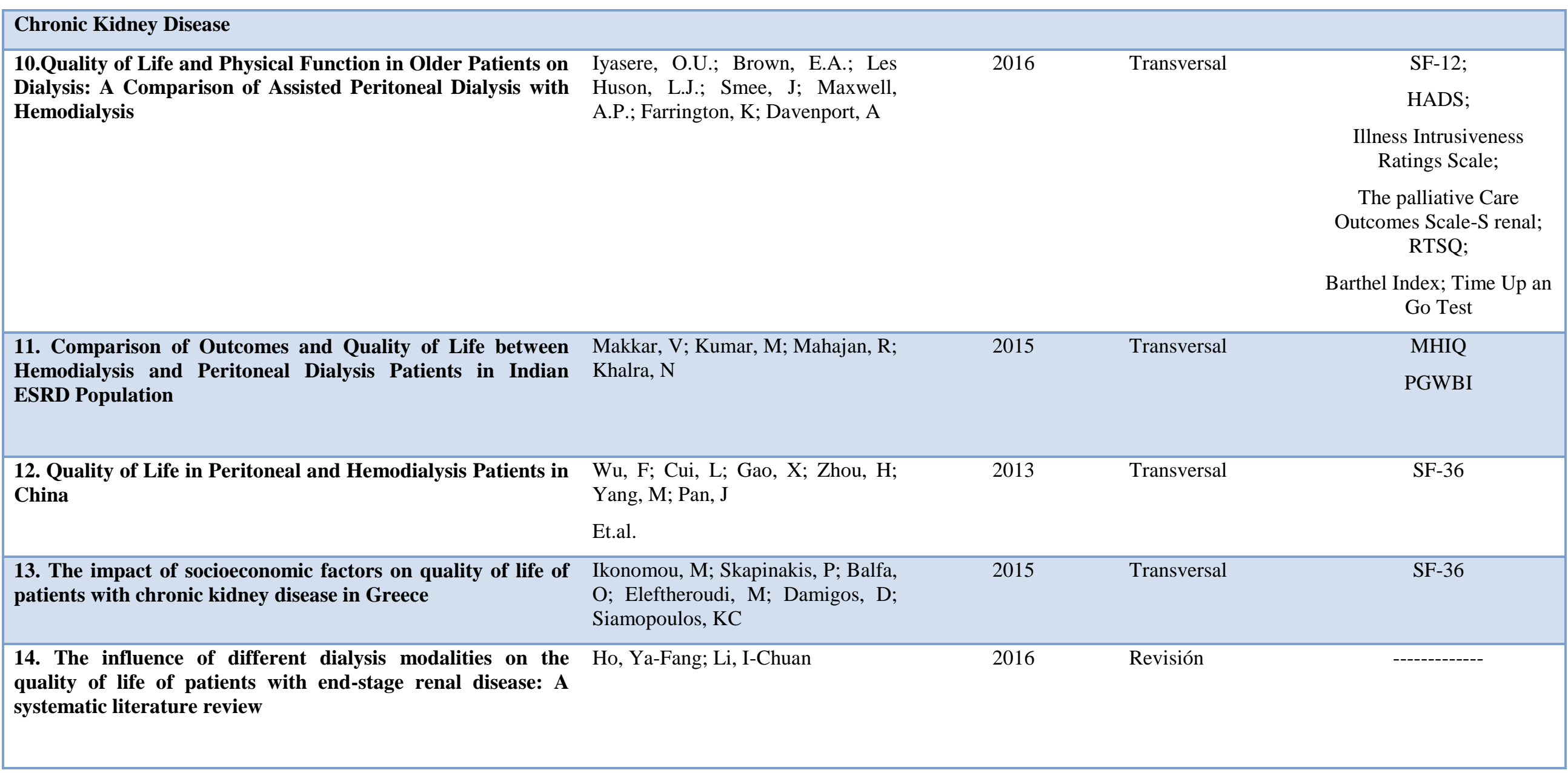


1.Quality of life in chronic haemodialysis and peritoneal dialysis patients in Turkey and related factors
HD> puntuación subescala general de salud.

PD> puntuación subescala del bienestar.

PD obtiene de forma estadísticamente significativa mayor puntuación en todas las subescalas SF-36 en comparación con el grupo de HD.

\section{Quality of Life and Self-Efficacy in Three Dialysis Modalities: Incenter \\ Hemodialysis, Home Hemodialysis, and Home Peritoneal Dialysis}

3. Quality of life in hemodialysis and peritoneal dialysis patients in Saudi Arabia Los pacientes con PD pasan más tiempo de calidad y estaban más satisfechos que los pacientes con HD.

Mejorara en la QOL puede ser adquirido en ambos grupos, si los programas de ejercicios están adecuadamente diseñados e implantados para satisfacer las demandas y necesidades de los pacientes.

4. Quality of life and mental health in hemodialysis and peritoneal dialysis patients: the role of health beliefs

Demuestra la importancia de comprender las creencias de enfermedad y tratamiento de los pacientes con ERCA y la contribución de estas a la QOL.

5. Quality of life and emotional distress between patients on peritoneal dialysis versus community-based hemodialysis

Aunque los niveles de QOL eran equivalentes entre PD y HD, hemos observado efectos dispares en la depresión y la satisfacción con la salud física y cuidado. En el contexto de la creciente incidencia de la PD es necesario supervisar las necesidades y preocupaciones de los pacientes con PD con el fin de orientar las intervenciones y expandir los programas de PD para promover el bienestar psicosocial.

6. Quality of life in end stage renal disease patients

Contradicciones con los resultados de unos estudios y otros.

Es necesario utilizar herramientas cualitativas que dan mucha información valiosa. 
7. Quality of life in patients on chronic dialysis in South Africa: a comparative mixed methods study

8. Quality of life of chronic renal patients in peritoneal dialysis and hemodialysis

9. Dyadic Relationship and Quality of Life Patients with Chronic Kidney Disease

10.Quality of Life and Physical Function in Older Patients on Dialysis: A Comparison of Assisted Peritoneal Dialysis with Hemodialysis
En general los resultados de KDQOL SF 1.3. no fueron diferentes para las dos modalidades.

Los pacientes de PD fueron más sintomáticos y experimentaron más limitaciones relacionados con el tratamiento que los de HD.

Al tratar de estudiar lo que influye en la QOL de nuestros pacientes de diálisis tenemos que mirar más allá de los aspectos clínicos y entender sus contextos reales.

La gran mayoría $(81,1 \%)$ de los pacientes están en HD. En cuanto a la QOL las personas en PD es superior a la de los pacientes en HD en el dominio del dolor. En el resto de los dominios no hubo diferencias estadísticamente significativas.

El tipo de terapia de sustitución renal afecta a la percepción de la QOL de los individuos con ERCA.

Una mejor comprensión diádica implica una mejor comprensión de la QOL

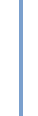
11. Comparison of Outcomes and Quality of Life between Hemodialysis and
Peritoneal Dialysis Patients in Indian ESRD Population
No hay diferencias en la QOL entre PD asistida y HD en pacientes de edad avanzada, a excepción de la satisfacción con el tratamiento, que es mayor en PD asistida que en HD.

Habría que plantearse la PD asistida para personas de edad avanzada desde el punto de vista de la QOL.

Debido al corto seguimiento y el pequeño tamaño de la muestra no hemos podido llegar a ninguna conclusión sobre el efecto a largo plazo de la modalidad de diálisis en la supervivencia y necesitamos estudios más amplios y de largo plazo para la misma en el contexto de la India.

Pacientes con mayor apoyo social podrían optar por la PD. Pacientes menos independientes podrían optar por HD.

12. Quality of Life in Peritoneal and Hemodialysis Patients in China 
13. The impact of socioeconomic factors on quality of life of patients with chronic kidney disease in Greece

Todo el sistema de cuidado (doctores, enfermeras, trabajadores sociales, familia, comunidad) debe ser consciente del problema, evaluar la QOL periódicamente y desarrollar intervenciones con el fin de mejorar su QOL.

14. The influence of different dialysis modalities on the quality of life of patients with end-stage renal disease: A systematic literature review
No se encontraron diferencias estadísticamente significativas.

Los diferentes síntomas de la enfermedad de los pacientes son factores importantes a tener cuenta por parte de los profesionales de la salud a la hora de aconsejar un tipo de terapia u otra. 
Calidad de vida en pacientes con ERCA en tratamiento con hemodiálisis vs diálisis peritoneal. Revisión integradora. 
Calidad de vida en pacientes con ERCA en tratamiento con hemodiálisis vs diálisis peritoneal. Revisión integradora.

\subsection{Evaluación de la calidad metodológica.}

Los estudios incluidos fueron evaluados a través de dos herramientas metodológicas. Las revisiones incluidas en los estudios escogidos, se evaluaron a través de la herramienta de lectura crítica CASPe, mientras que los estudios observacionales se evaluaron a través de STROBE, que consta de una lista de verificación de 22 puntos.

CASPe es una herramienta de lectura crítica que sirve para evaluar la calidad metodológica de una serie de estudios, entre los que se encuentran las revisiones sistemáticas. En el caso de las revisiones este instrumento tiene 10 preguntas a contestar. $^{(16)}$

La lista de verificación de STROBE fue una iniciativa internacional, en colaboración de epidemiólogos, especialistas en metodología, estadísticos, investigadores y editores de revistas que participan en la realización y difusión de estudios de observación, con el objetivo común de mejorar la comunicación de estudios observacionales en epidemiología. ${ }^{(17)}$

De los 12 estudios transversales, 7 cumplieron todos los puntos del check-list de STROBE. ${ }^{(18)(19)(20)(21)(22)(23)(24)}$ El resto de los estudios transversales no cumplían algún punto de la lista.. Los puntos no cumplidos fueron el $17^{(25)(26)(27)}$ y el $22^{(25)(26)(28)(29)}$. Gráfica 4, Tabla 10 y 11 . De los estudios que si que cumplían el punto 22 , $5^{(18)(21)(23)(24)(27)}$ declararon no presentar ningún conflicto de intereses y $3^{(19)(20)(22)}$ declararon que fueron financiados por fondos externos.

Ambas revisiones ${ }^{(14)(30)}$ del presente estudio pasaron todos los ítems de la herramienta CASPe a excepción del punto 7 , puesto que no se encontraron intervalos de confianza. 
Calidad de vida en pacientes con ERCA en tratamiento con hemodiálisis vs diálisis peritoneal. Revisión integradora.

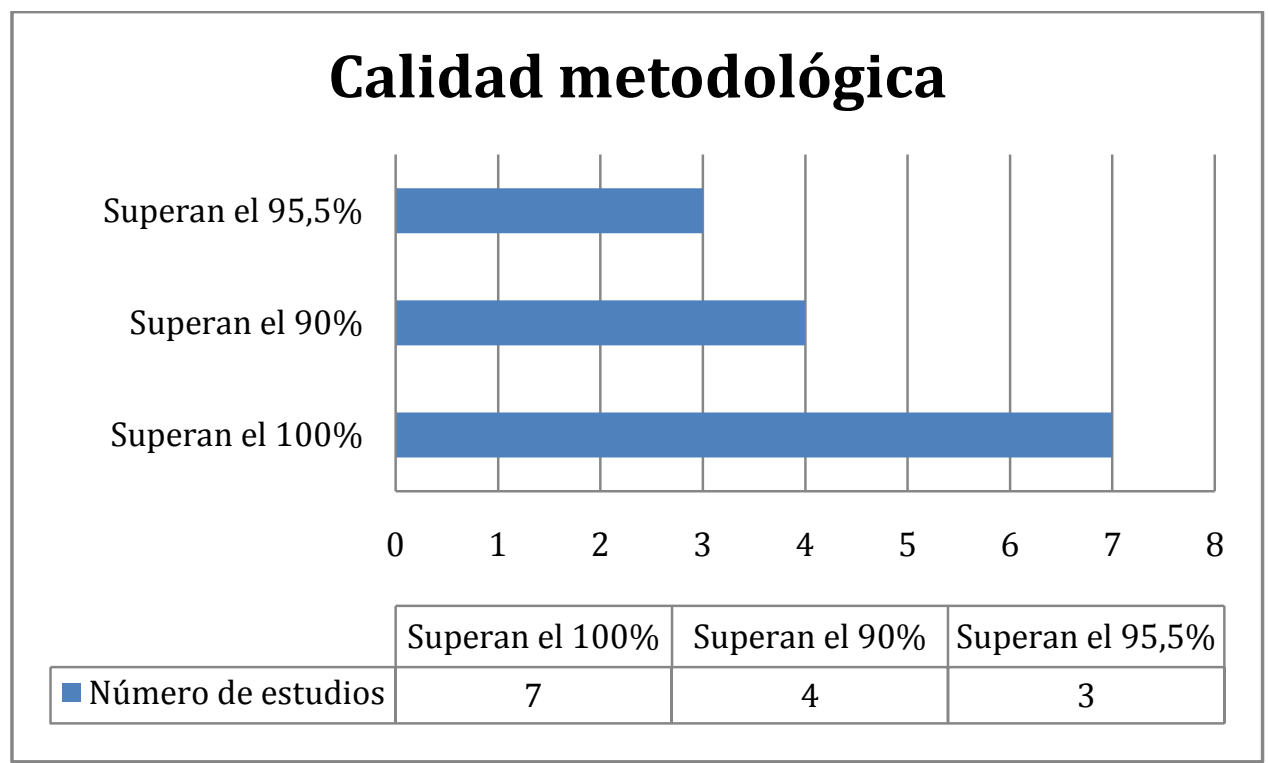

Gráfica 4. Porcentaje de Calidad Medtodológica. 
Tabla 10. Resultados check-list STROBE.

\begin{tabular}{|c|c|c|c|c|c|c|c|c|c|c|c|c|}
\hline STROBE & $\begin{array}{c}\text { Estudio } \\
1\end{array}$ & $\begin{array}{c}\text { Estudio } \\
2\end{array}$ & $\begin{array}{c}\text { Estudio } \\
3\end{array}$ & $\begin{array}{c}\text { Estudio } \\
4\end{array}$ & $\begin{array}{c}\text { Estudio } \\
5\end{array}$ & $\begin{array}{c}\text { Estudio } \\
7\end{array}$ & $\begin{array}{c}\text { Estudio } \\
8\end{array}$ & $\begin{array}{c}\text { Estudio } \\
9\end{array}$ & $\begin{array}{c}\text { Estudio } \\
10\end{array}$ & $\begin{array}{c}\text { Estudio } \\
11\end{array}$ & $\begin{array}{c}\text { Estudio } \\
12\end{array}$ & $\begin{array}{c}\text { Estudio } \\
13\end{array}$ \\
\hline Punto 1 & si & si & si & si & si & si & si & si & si & si & si & si \\
\hline Punto 3 & si & si & si & si & si & si & si & si & si & si & si & si \\
\hline Punto 4 & si & si & si & si & si & si & si & si & si & si & si & si \\
\hline Punto 5 & si & si & si & si & si & si & si & si & si & si & si & si \\
\hline Punto 6 & si & si & si & si & si & si & si & si & si & si & si & si \\
\hline Punto 7 & si & si & si & si & si & si & si & si & si & si & si & si \\
\hline Punto 8 & si & si & si & si & si & si & si & si & si & si & si & si \\
\hline Punto 9 & si & si & si & si & si & si & si & si & si & si & si & si \\
\hline Punto 10 & si & si & si & si & si & si & si & si & si & si & si & si \\
\hline Punto 11 & si & si & si & si & si & si & si & si & si & si & si & si \\
\hline Punto 12 & si & si & si & si & si & si & si & si & si & si & si & si \\
\hline Punto 13 & si & si & si & si & si & si & si & si & si & si & si & si \\
\hline Punto 14 & si & si & si & si & si & si & si & si & si & si & si & si \\
\hline Punto 15 & si & si & si & si & si & si & si & si & si & si & si & si \\
\hline Punto 16 & si & si & si & si & si & si & si & si & si & si & si & si \\
\hline Punto 17 & si & si & si & NO & si & si & si & NO & si & NO & si & si \\
\hline Punto 18 & si & si & si & si & si & si & si & si & si & si & si & si \\
\hline Punto 19 & si & si & si & si & si & si & si & si & si & si & si & si \\
\hline Punto 20 & si & si & si & si & si & si & si & si & si & si & si & si \\
\hline Punto 21 & si & si & si & si & si & si & si & si & si & si & si & si \\
\hline Punto 22 & NO & si & si & NO & si & si & NO & NO & si & si & si & si \\
\hline
\end{tabular}


Tabla 11. Resultados herramienta lectura crítica CASPe.

\begin{tabular}{|c|c|c|}
\hline $\begin{array}{l}\text { CASPe } \\
\text { Revisiones }\end{array}$ & 6. Quality of life in end stage renal disease patients & $\begin{array}{l}\text { 14. The influence of different dialysis modalities on the } \\
\text { quality of life of patients with end-stage renal disease: A } \\
\text { systematic literature review }\end{array}$ \\
\hline Pregunta 1 & SÍ & SÍ \\
\hline Pregunta 2 & SÍ & SÍ \\
\hline Pregunta 3 & SÍ & SÍ \\
\hline Pregunta 4 & SÍ & SÍ \\
\hline Pregunta 5 & SÍ & SÍ \\
\hline Pregunta 6 & $\begin{array}{l}\text { Hay una contradicción entre los resultados de los estudios. } \\
\text { Hay que plantear que es más importante el tipo de tratamiento } \\
\text { o el lugar en el que se lleva a cabo. }\end{array}$ & $\begin{array}{l}\text { No se encontraron diferencias estadísticamente significativas, } \\
\text { pero la DP obtiene mayor porcentaje en las puntuaciones de } \\
\text { calidad de vida. La razón podría ser posiblemente la } \\
\text { independencia a la hora de administrar el tratamiento y la mayor } \\
\text { autonomía que proporciona. }\end{array}$ \\
\hline Pregunta 7 & No aparece ningún intervalo de confianza. & No aparece ningún intervalo de confianza \\
\hline Pregunta 8 & SÍ & SÍ \\
\hline Pregunta 9 & SÍ & SÍ \\
\hline Pregunta 10 & SÍ & SÍ \\
\hline
\end{tabular}


Calidad de vida en pacientes con ERCA en tratamiento con hemodiálisis vs diálisis peritoneal. Revisión integradora. 
Calidad de vida en pacientes con ERCA en tratamiento con hemodiálisis vs diálisis peritoneal. Revisión integradora.

\section{Limitaciones.}

La principal limitación de esta revisión es la poca variedad de estudios encontrados, puesto que solo hay dos revisiones de la bibliografía y el resto son estudios observacionales descriptivos de tipo transversal. Debido al tipo de población a estudio y la variable a evaluar (QOL), es difícil que se realicen estudios de tipo experimental. Para realizar una mejor valoración sería necesario utilizar herramientas cualitativas, puesto que dan mucha información en este tipo de estudios, además de estudios de tipo longitudinal, para observar los cambios de la QOL en el tiempo. 
Calidad de vida en pacientes con ERCA en tratamiento con hemodiálisis vs diálisis peritoneal. Revisión integradora.

\section{Discusión.}

La calidad de vida es un factor transcendental para los pacientes con ERCA. Para medirla existe una serie de cuestionarios validados internacionalmente y de amplio uso que han sido aplicados en los estudios recopilados en esta revisión. Los cuestionarios más ampliamente utilizados son el cuestionario de calidad de vida específico para la enfermedad renal $(\mathrm{KDQOL}-\mathrm{SF})^{(18)(19)(21)}$ y sus versiones cortas $\left(\mathrm{SF}-36\right.$ y SF-12) ${ }^{(20)(22)(23)(24)(28)(29)}$ y el cuestionario de calidad de vida de la OMS (WHOQOLBREF) $^{(20)(25)(26)}$.

La calidad de vida es una media multidimensional y a la hora de valorarla, se tiene en cuenta tanto el componente físico como el mental.

Las principales variables sociodemográficas y clínicas que muestran una relación estadística significativa para la población de estudiocon la QOL según los estudios recopilados son, la edad ${ }^{(19)(27)(28)}$,el sexo ${ }^{(19)(28)}$, la educación ${ }^{(28)}$, factores sociales y económicos $^{(21)(24)}$, los niveles séricos de prealbúmina ${ }^{(18)(29)}$, la presencia de comorbilidades $^{(27)(28)}$, la capacidad de llevar a cabo el tratamiento ${ }^{(27)}$ y el tiempo de duración de la diálisisis(19)(30).

Algunos de los autores, referencian la gran importancia del apoyo social tanto de familiares, compañeros y amigos, como del personal sanitario. ${ }^{(21)(27)}$ Según Makkar, $V$. et. al. los pacientes con mayor apoyo social serían candidatos para optar por el tratamiento con PD, y aquellos pacientes con menor independencia sería más recomendable el tratamiento con $\mathrm{HD}{ }^{(27)}$

En relación a lapercepción de la vida sexual, en ambos tipos de tratamiento, los pacientes manifestaron una disminución del deseo sexual ${ }^{(21)}$, además de una disfunción eréctil en los pacientes masculinos tratados con HD. ${ }^{(21)}$ Los pacientes en PD afirman que éste se ve afectado por la imagen corporal, debido al catéter peritoneal, aunque según los resultados obtenidos por Wright, LS. et.al. los pacientes en PD manifestaron mayor disfunción eréctil. ${ }^{(18)}$

Según los resultados obtenidos por Griva, K. et.al.yTheofilou, P.et.al.existen tasas de depresión sustancialmente mayores en los pacientes de PD. ${ }^{(20)(25)}$ Sin embargo en 
Calidad de vida en pacientes con ERCA en tratamiento con hemodiálisis vs diálisis peritoneal. Revisión integradora.

cuanto a los síntomas de ansiedad o tasas de posibles casos de ansiedad, no hay diferencias entre modalidades de diálisis. ${ }^{(20)}$ Estos hallazgos, entran en controversia con los obtenidos por Makkar, V.et.al., que afirman que los pacientes en HD tienen mayor tasas de depresión y suicidio. En las tasas de suicidio se incluyen las violaciones de la dieta ${ }^{(27)}$, puesto que un incumplimiento excesivo de la dieta produce perjuicios para la salud de estos pacientes agravando en gran medida su estado.

La tasa de reingreso hospitalario es otra variable que se muestra decisiva en la percepción de la calidad de vida por parte de los pacientes. En el tratamiento con PD la tasa de hospitalización es mayor que en $\mathrm{HD}$, debido a los casos reincidentes de peritonitis que se producen en este grupo. ${ }^{(27)(28)(29)}$ y relacionada con una falta de asepsia en la manipulación del catéter peritoneal en el proceso de los recambios.

En cuanto a la capacidad de trabajar,según Tannor, EK. et.al. y Oren, Beseyet.al.los pacientes en PD tienen más posibilidad de continuar con su trabajo, y hay una mayor tasa de desempleo en los pacientes tratados con HD.(21) ${ }^{(28)}$ Aunque Tannor, EK. et.al. puntualiza que a pesar de que es cierto que los paciente en PD tienen mejor puntuaciones en la continuidad de trabajo también reportaron dificultades para encontrar trabajo y poder realizar intercambios de bolsa en el puesto de trabajo. ${ }^{(21)}$

En la calidad del sueño medida a través del subapartado específico del cuestionario KDQOL-SF, los pacientes en PD suelen tener menor puntuación que los pacientes de $\mathrm{HD}^{(18)(21)}$, esto es razonables debido a que el tratamiento de PD suele ser nocturno, y los intercambios se realizan mientras el paciente duerme.

Un gran número de estudios ${ }^{(14)(18)(21)(22)(23)(29)}$, presentan entre sus conclusiones que los pacientes tratados con PD obtuvieron mayor puntuación en gran parte de las escalas de los cuestionarios, pero estos resultados no mostraron una significación estadística concluyente. Hay que tener en cuenta, que los pacientes en PD suelen ser más jóvenes y presentar menos comorbilidades que los pacientes en HD, donde suelen encontrarse pacientes más longevos con una autonomíalimitada para llevar el tratamiento domiciliario, además de la mayor independencia y el poder autogestionar el tratamiento. ${ }^{(14)}$

Según Iyasere, OU. et al, que compara la QOL de los pacientes de edad avanzada en ambos tipos de diálisis estudiados (HD y PD), en el único apartado en el que hay una 
Calidad de vida en pacientes con ERCA en tratamiento con hemodiálisis vs diálisis peritoneal. Revisión integradora.

diferencia significativa es con la satisfacción del tratamiento, siendo mayor en la PD asistida. Como se menciona en el párrafo anterior, los pacientes de mayor edad suelen ser tratados en HD, pero se debería plantear como alternativa para estas personas, el tratamiento con PD asistida desde el punto de vista de la QOL. ${ }^{(22)}$

A la hora de aconsejar un tratamiento $u$ otro, es de gran importancia que los profesionales de la salud tengan en cuenta además los síntomas de la enfermedad que presentan los pacientes, la anemia y los niveles bajos de albúmina. En pacientes con anemia grave sería más adecuado recibir PD y la HD sería más adecuada en los pacientes con bajos niveles de albúmina. ${ }^{(14)}$

Por último, Joshi, VD.concluye su revisión cuestionando que igual habría que tener en cuenta si lo que realmente afecta a la percepción de la calidad de vida es el tipo de tratamiento, o la duración de este, puesto que una gran parte de los pacientes que en un principio inician el tratamiento de sustitución renal en PD, acaban siendo tratados con HD. ${ }^{(30)}$ 
Calidad de vida en pacientes con ERCA en tratamiento con hemodiálisis vs diálisis peritoneal. Revisión integradora. 
Calidad de vida en pacientes con ERCA en tratamiento con hemodiálisis vs diálisis peritoneal. Revisión integradora.

\section{Conclusiones.}

No encontramos evidencias científicas en la literatura actual que nos permita afirmar categóricamenteque un tipo de tratamiento de sustitución renal resulte mejor que otro, en relación al impacto que produce el mismo sobre la calidad de vida.

Los profesionales sanitarios deben analizar cada paciente individualmente y las variables que pueden influir a la hora de prescribir un tipo de tratamiento u otro. Es necesario que realicen periódicamente una evaluación del paciente para controlar si se han modificado sus variables y necesidades, y adaptar el tratamiento a estas. Los pacientes que padezcan anemia grave sería más recomendable un tratamiento con $\mathrm{PD}$, y aquellos pacientes que presenten bajos niveles de albúmina deberían ser tratados con HD.

Las principales variables sociodemográficas y clínicas que muestran una relación estadística significativa para la población de estudio con la QOL según los estudios recopilados son, la edad, el sexo, la educación, factores sociales y económicos, los niveles séricos de prealbúmina, la presencia de comorbilidades, la capacidad de llevar a cabo el tratamiento y el tiempo de duración de la diálisis.

Para concluir, considero que es necesario continuar con más estudios de investigación en este campo de actuacióny así poder observar si hay una variación de la percepción de la calidad de vida de los pacientes con el paso de los años en tratamiento de sustitución de la función renal, por lo que sería conveniente el diseño de estudios de cohortes de tipo longitudinal. 
Calidad de vida en pacientes con ERCA en tratamiento con hemodiálisis vs diálisis peritoneal. Revisión integradora. 
Calidad de vida en pacientes con ERCA en tratamiento con hemodiálisis vs diálisis peritoneal. Revisión integradora.

\section{Referencias bibliográficas.}

1. OPS OMS | Enfermedad crónica del riñón [Internet]. [cited 2017 Mar 1]. Available from: http://www.paho.org/hq/index.php?option=com_topics\&view=article\&id=349 \&Itemid=40937\&lang=es

2. Lorenzo Sellarés V. Enfermedad renal crónica [Internet]. [cited 2017 Mar 1]. Available from: http://www.revistanefrologia.com/es-publicacion-nefrologiaarticulo-enfermedad-renal-cronica-XX342164212000426

3. Martínez-Castelao A, Górriz J, Bover J. Documento de consenso para la detección y manejo de la enfermedad renal crónica. Atención Primaria [Internet]. 2014 [cited 2017 Mar 1]; Available from: http://www.sciencedirect.com/science/article/pii/S021265671400273X

4. Urrutia CN, Mella PO. Función renal y factores asociados en el desarrollo de la enfermedad renal crónica en adultos. Rev Cuba [Internet]. 2014 [cited 2017 Mar 1]; Available from: http://scielo.sld.cu/scielo.php?script=sci_arttext\&pid=S086403192014000400004

5. Etapas de la enfermedad renal crónica (ERC) | Fresenius Medical Care [Internet]. [cited 2017 Mar 1]. Available from: http://www.ultracaredialysis.com/es-ES/kidney-disease/chronic/ckd-stages.aspx\#tab-stages-ofkidney-disease-4

6. Flores J, Alvo M, Borja H, Morales J, Vega J. Enfermedad renal crónica: Clasificación, identificación, manejo y complicaciones. médica de Chile [Internet]. 2009 [cited 2017 Mar 1]; Available from: http://www.scielo.cl/scielo.php?pid=S003498872009000100026\&script=sci_arttext

7. Jiménez AG, Hernández OM. Enfermedad renal crónica en el adulto mayor. Rev Med (Puebla) [Internet]. 2013 [cited 2017 Mar 1]; Available from: http://scielo.sld.cu/scielo.php?script=sci_arttext\&pid=S168418242013000400001

8. Edici RDELA, Salud G, Epidem SE. Informe del Registro de Enfermos Renales de la Comunitat Valenciana. 2015;1-44.

9. Tipos de tratamiento [Internet]. [cited 2017 May 12]. Available from: https://www.diaverum.com/es-ES/Informacion-sobre-los-rinones/Tipos-detratamiento/

10. Tratamientos: hemodiálisis y diálisis peritoneal |: : FRIAT / Fundación Renal:: [Internet]. [cited 2017 Mar 1]. Available from: https://www.friat.es/la-enfermedad-renal/insuficiencia-renal-

cronica/tratamientos-hemodialisis-y-dialisis-peritoneal/ 
Calidad de vida en pacientes con ERCA en tratamiento con hemodiálisis vs diálisis peritoneal. Revisión integradora.

11. Hemodiálisis [Internet]. [cited 2017 May 12]. Available from: https://www.diaverum.com/es-ES/Tratamiento/Hemodialisis/

12. Diálisis peritoneal [Internet]. [cited 2017 May 12]. Available from: https://www.diaverum.com/es-ES/Tratamiento/Dialisis-peritoneal/

13. Rebollo-Rubio A, Morales-Asencio JM, Pons-Raventos ME, MansillaFrancisco JJ. Revisión de estudios sobre calidad de vida relacionada con la salud en la enfermedad renal crónica avanzada en España. Nefrologia. 2015;35(1):92-109.

14. Ho Y-F, Li I-C. The influence of different dialysis modalities on the quality of life of patients with end-stage renal disease: A systematic literature review. Psychol Health [Internet]. 2016 Dec 7 [cited 2017 Apr 2];31(12):1435-65. Available from: https://www.tandfonline.com/doi/full/10.1080/08870446.2016.1226307

15. Perestelo-Pérez L. Standards on how to develop and report systematic reviews in Psychology and Health. Int J Clin Heal Psychol [Internet]. 2013 Jan [cited 2017 May 12];13(1):49-57. Available from: http://linkinghub.elsevier.com/retrieve/pii/S1697260013700073

16. Instrumentos para la lectura crítica | CASPe [Internet]. [cited 2017 May 14]. Available from: http://www.redcaspe.org/herramientas/instrumentos

17. STROBE Statement: Home [Internet]. [cited 2017 May 14]. Available from: https://www.strobe-statement.org/index.php?id=strobe-home

18. Wright L, Wilson L. Quality of life and self-efficacy in three dialysis modalities: incenter hemodialysis, home hemodialysis, and home peritoneal dialysis. Nephrol Nurs J [Internet]. 2015 [cited 2017 May 12]; Available from: http://search.proquest.com/openview/e1222ca947511631bd099ead56032e2b/1 ?pq-origsite $=$ gscholar $\& \mathrm{cbl}=45638$

19. Wakeel, Jamal Al; Harbi, Ali Al; Bayoumi, Magda; Al-Suwaida, Karaem; Ghonaim MA et al. Quality of life in hemodialysis and peritoneal dialysis patients in Saudi Arabia. 2012 [cited 2017 May 14]; Available from: http://search.proquest.com/healthcomplete/docview/1443433275/9211126C04 8D4EC5PQ/1? accountid $=15297$

20. Griva K, Yu Z, Chan S, Krisnasamy T, Yamin RBA, Zakaria FB, et al. Age is not a contraindication to home-based dialysis - Quality-of-Life outcomes favour older patients on peritoneal dialysis regimes relative to younger patients. J Adv Nurs [Internet]. 2014 Aug [cited 2017 Jan 24];70(8):1902-14. Available from: http://www.ncbi.nlm.nih.gov/pubmed/24495288

21. Tannor EK, Archer E, Kapembwa K, van Schalkwyk SC, Davids MR. Quality of life in patients on chronic dialysis in South Africa: a comparative mixed methods study. BMC Nephrol [Internet]. 2017 Jan 5 [cited 2017 Jan 24];18(1):4. Available from: http://bmcnephrol.biomedcentral.com/articles/10.1186/s12882-016-0425-1 
Calidad de vida en pacientes con ERCA en tratamiento con hemodiálisis vs diálisis peritoneal. Revisión integradora.

22. Iyasere OU, Brown EA, Johansson L, Huson L, Smee J, Maxwell AP, et al. Quality of Life and Physical Function in Older Patients on Dialysis: A Comparison of Assisted Peritoneal Dialysis with Hemodialysis. Clin J Am Soc Nephrol [Internet]. 2016 Mar 7 [cited 2017 Jan 24];11(3):423-30. Available from: http://cjasn.asnjournals.org/cgi/doi/10.2215/CJN.01050115

23. Wu F, Cui L, Gao X, Zhou H, Yang M, Pan J, et al. Quality of life in peritoneal and hemodialysis patients in China. Ren Fail [Internet]. 2013;35(4):456-9. Available from: http://www.ncbi.nlm.nih.gov/pubmed/23406057

24. Ikonomou M, Skapinakis $\mathrm{P}$, Balafa O, Eleftheroudi M, Damigos D, Siamopoulos KC. THE IMPACT OF SOCIOECONOMIC FACTORS ON QUALITY OF LIFE OF PATIENTS WITH CHRONIC KIDNEY DISEASE IN GREECE. J Ren Care [Internet]. 2015 Dec [cited 2017 May 14];41(4):239-46. Available from: http://doi.wiley.com/10.1111/jorc.12132

25. Theofilou P. Quality of life and mental health in hemodialysis and peritoneal dialysis patients: the role of health beliefs. [cited 2017 May 14]; Available from:

http://search.proquest.com/healthcomplete/docview/914866492/3A7F2B73F9 934177PQ/2?accountid $=15297$

26. Barata NERRC. Dyadic Relationship and Quality of Life Patients with Chronic Kidney Disease. J Bras Nefrol [Internet]. 2015 [cited 2017 Jan 24];37(3):315-22. Available from: http://www.gnresearch.org/doi/10.5935/0101-2800.20150051

27. Makkar V, Kumar M, Mahajan R, Khaira NS. Comparison of Outcomes and Quality of Life between Hemodialysis and Peritoneal Dialysis Patients in Indian ESRD Population. J Clin Diagn Res [Internet]. 2015 Mar [cited 2017 Jan 24];9(3):OC28-31. Available from: http://jcdr.net/article_fulltext.asp?issn=0973$709 x \&$ year $=2015 \&$ volume $=9 \&$ issue $=3 \&$ page $=0 C 28 \&$ issn $=0973-$ $709 x \& i d=5709$

28. Oren B, Enç N. Quality of life in chronic haemodialysis and peritoneal dialysis patients in Turkey and related factors. Int J Nurs Pract [Internet]. 2013 Dec [cited 2017 Jan 24];19(6):547-56. Available from: http://www.ncbi.nlm.nih.gov/pubmed/24330205

29. Ramos ECC, Santos I da S dos, Zanini R de V, Ramos JMG. Quality of life of chronic renal patients in peritoneal dialysis and hemodialysis. J Bras Nefrol [Internet]. 2015 [cited 2017 Jan 24];37(3):297-305. Available from: http://www.gnresearch.org/doi/10.5935/0101-2800.20150049

30. Joshi VD. Quality of life in end stage renal disease patients. World J Nephrol [Internet]. 2014 Nov 6 [cited 2017 Jan 24];3(4):308-16. Available from: http://www.wjgnet.com/2220-6124/full/v3/i4/308.htm 
Calidad de vida en pacientes con ERCA en tratamiento con hemodiálisis vs diálisis peritoneal. Revisión integradora. 
Calidad de vida en pacientes con ERCA en tratamiento con hemodiálisis vs diálisis peritoneal. Revisión integradora.

\section{Anexos}

Anexo 1. Herramienta de calidad metodológica CASPe.

\section{A/ ¿Los resultados de la revisión son válidos?}

\section{Preguntas "de eliminación"}

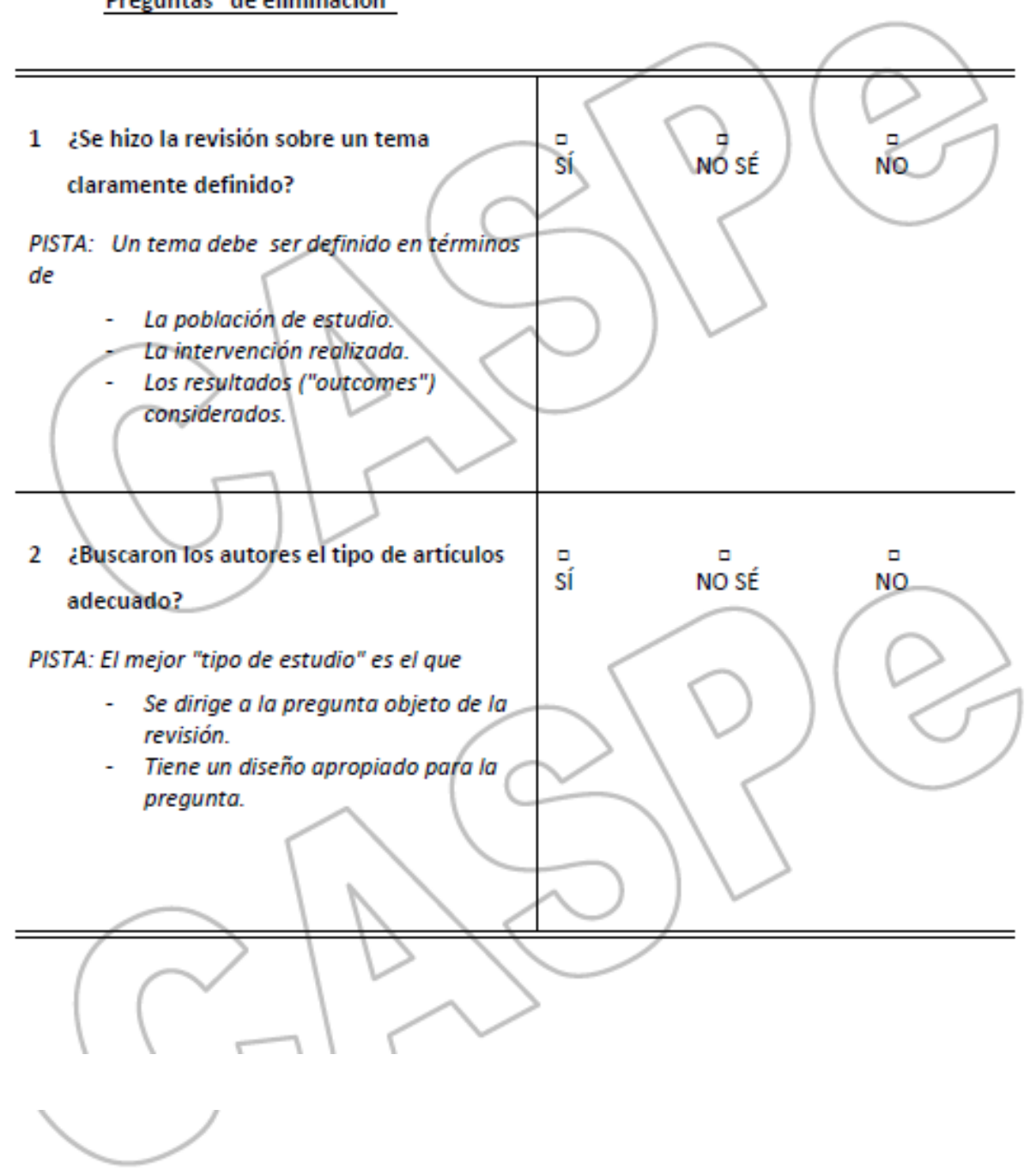

¿Merece la pena continuar? 
Calidad de vida en pacientes con ERCA en tratamiento con hemodiálisis vs diálisis peritoneal. Revisión integradora.

Preguntas detalladas

3 ¿Crees que estaban incluidos los estudios importantes y pertinentes?

PISTA: Busca

- Qué bases de datos bibliográficas se han usado.

- Seguimiento de las referencias.

- Contacto personal con expertos.

- Búsqueda de estudios no publicados.

Búsqueda de estudios en idiomas distintos del inglés.

4 Crees que los autores de la revisión han hecho suficiente esfuerzo para valorar la calidad de los estudios incluidos?

PISTA: Los autores necesitan considerar el rigor de los estudios que han identificado. La falta de rigor puede afectar al resultado de los estudios (NNo es oro todo lo que reluce" El Mercoder de Venecia. Acto II)

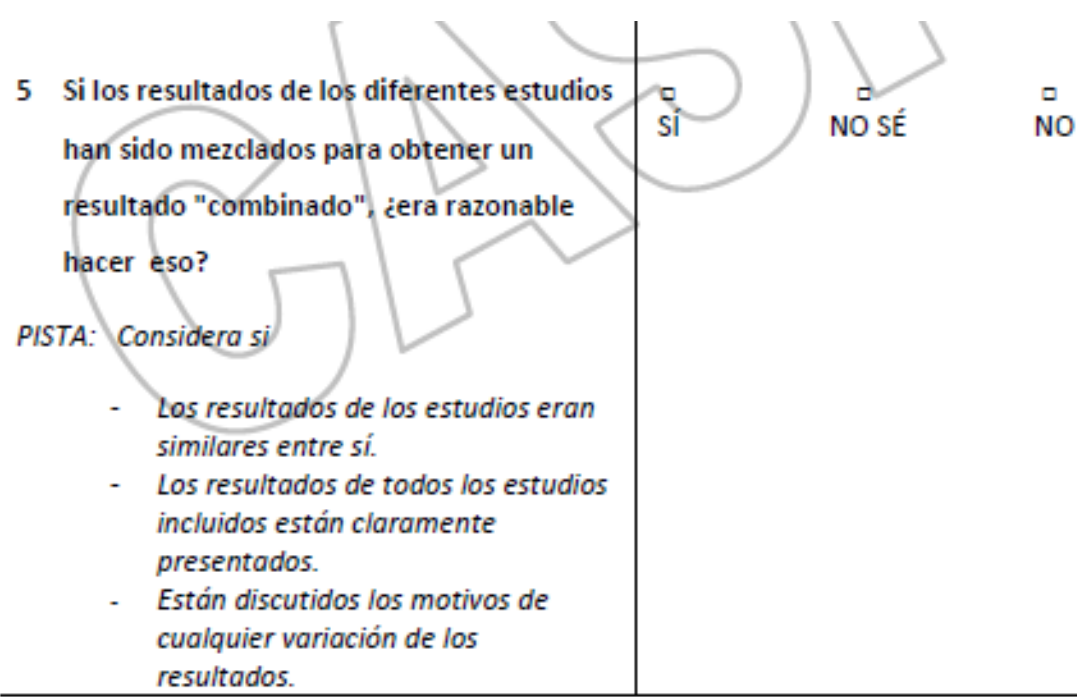


Calidad de vida en pacientes con ERCA en tratamiento con hemodiálisis vs diálisis peritoneal. Revisión integradora.

\section{B/ ¿Cuáles son los resultados?}

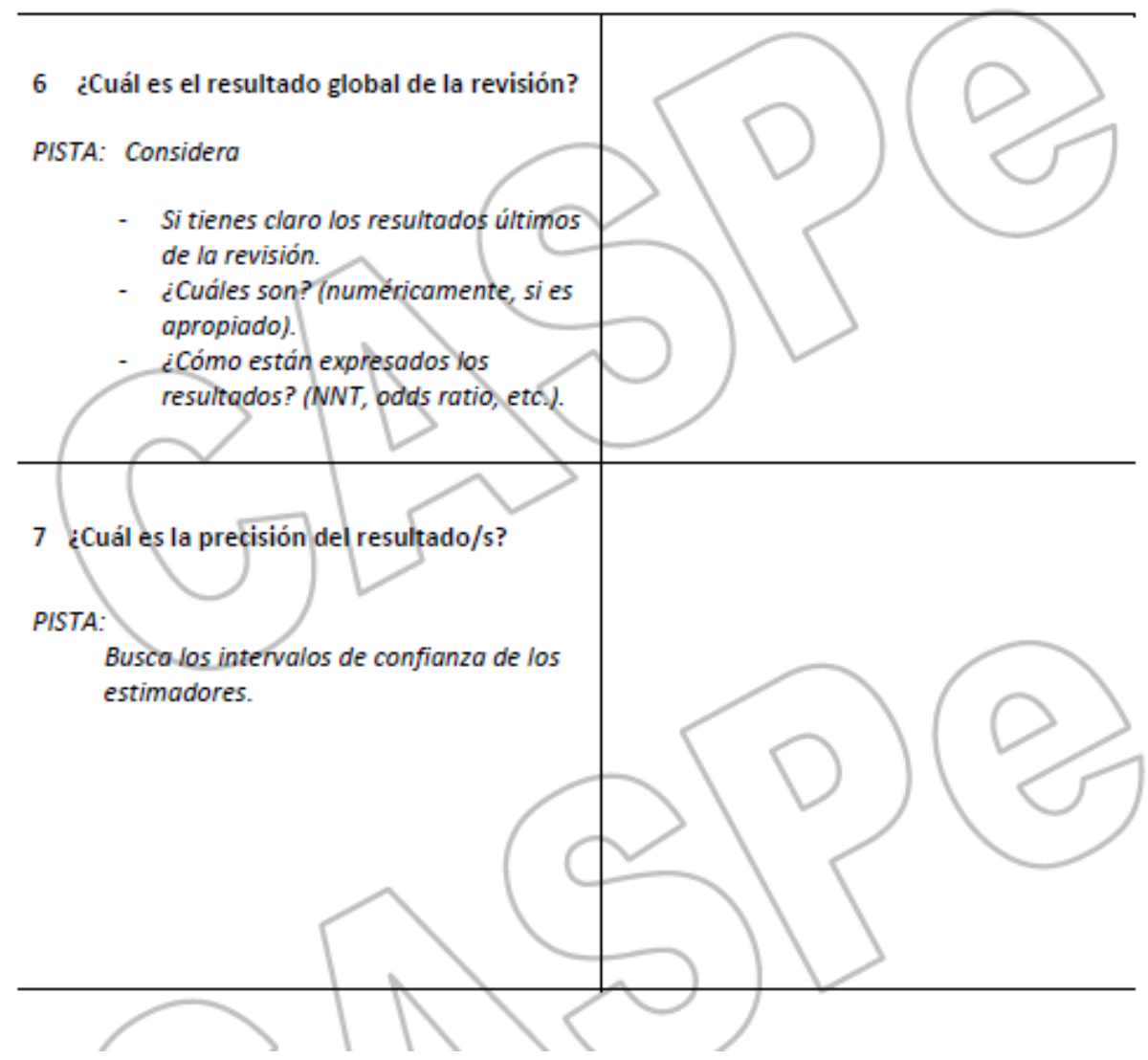


Calidad de vida en pacientes con ERCA en tratamiento con hemodiálisis vs diálisis peritoneal. Revisión integradora.

\section{C/¿Son los resultados aplicables en tu medio?}

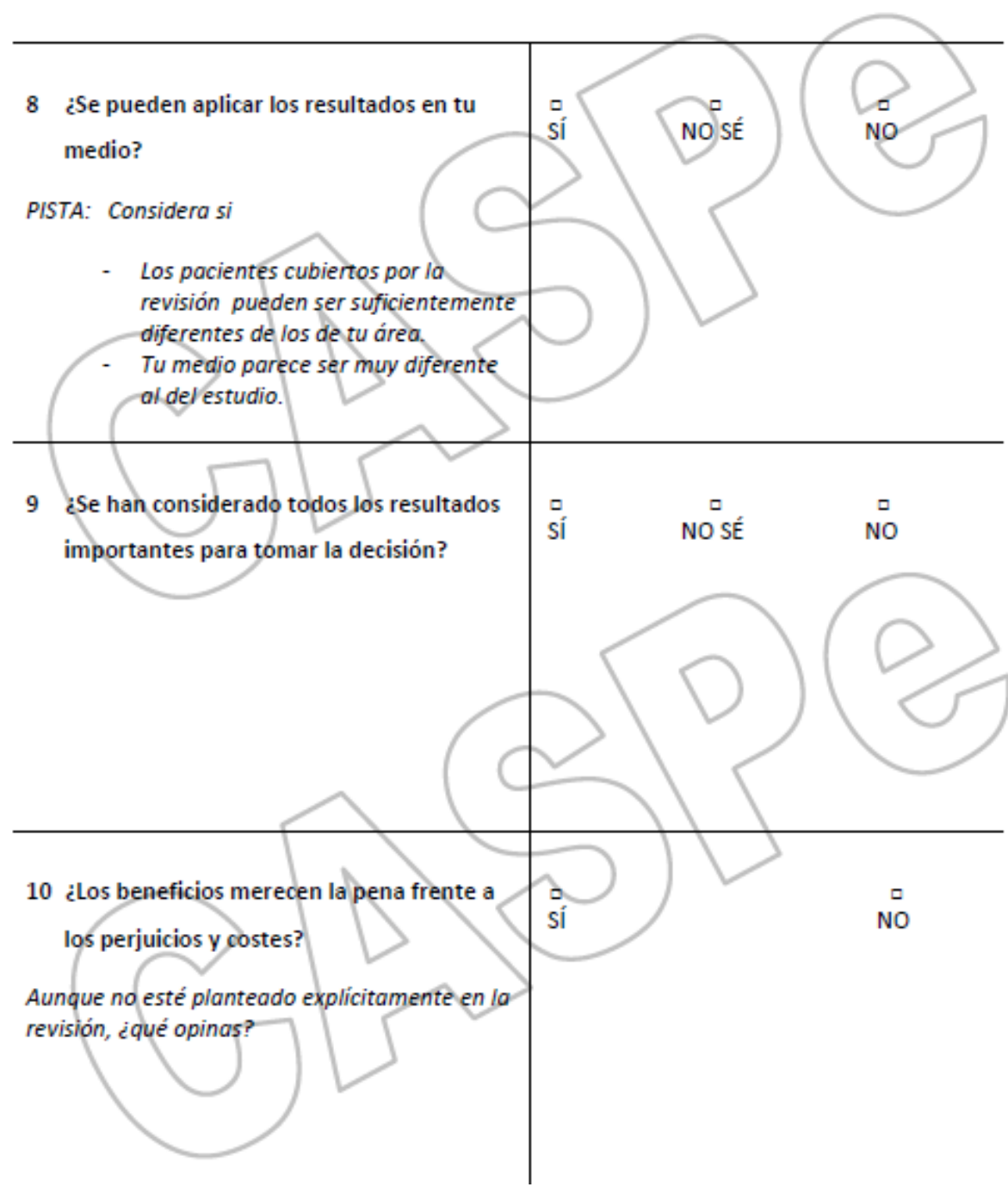


Calidad de vida en pacientes con ERCA en tratamiento con hemodiálisis vs diálisis peritoneal. Revisión integradora.

Anexo 2. Herramienta de calidad metodológica STROBE.

von Elm E et al. Declaración de la Iniciativa STROBE (Strengthening the Reporting of Observational studies in Epidemiology): directrices para la comunicación de estudios observacionales

\begin{tabular}{|c|c|c|}
\hline Titulo y resumen & Punto & Recomendación \\
\hline & 1 & $\begin{array}{l}\text { (a) Indique, en el titulo o en el resumen, el disento del estudio con un término habitual } \\
\text { (b) Proporcione en el resumen una sinopsis informativa y equilibrada de lo que se ha hecho y lo que se ha encontrado }\end{array}$ \\
\hline \multicolumn{3}{|l|}{ Introducción } \\
\hline $\begin{array}{l}\text { Contexto/fundamentos } \\
\text { Objetivos }\end{array}$ & $\begin{array}{l}2 \\
3\end{array}$ & $\begin{array}{l}\text { Explique las razones y el fundamento cientfificos de la investigación que se comunica } \\
\text { Indique los objetivos especificos, incluida cualquier hipótesis preespecificada }\end{array}$ \\
\hline \multicolumn{3}{|l|}{ Métodos } \\
\hline Diseño del estudio & 4 & Presente al principio del documento los elementos clave del diseño del estudio \\
\hline Contexto & 5 & $\begin{array}{l}\text { Describa el marco, los lugares y las fechas relevantes, incluido los periodos de reclutamiento, exposición, seguimiento y } \\
\text { recogida de datos }\end{array}$ \\
\hline \multirow[t]{4}{*}{ Participantes } & 6 & $\begin{array}{l}\text { (a) Estudios de cohortes: proporcione los criterios de elegibilidad, asi como las fuentes y el método de selección de los } \\
\text { participantes. Especifique los métodos de seguimiento }\end{array}$ \\
\hline & & $\begin{array}{l}\text { Estudios de casos y controles: proporcione los criterios de elegibilidad así como las fuentes y el proceso diagnóstico de los } \\
\text { casos y el de selección de los controles. Proporcione las razones para la elección de casos y controles }\end{array}$ \\
\hline & & $\begin{array}{l}\text { Estudios transversales: proporcione los criterios de elegibilidad y las fuentes y métodos de selección de los participantes } \\
\text { (b) Estudios de cohortes: en los estudios apareados, proporcione los criterios para la formación de parejas y el número de } \\
\text { participantes con y sin exposición }\end{array}$ \\
\hline & & $\begin{array}{l}\text { Estudios de casos y controles: en los estudios apareados, proporcione los criterios para la formación de las parejas y el } \\
\text { número de controles por cada caso }\end{array}$ \\
\hline Variables & 7 & $\begin{array}{l}\text { Defina claramente todas las variables: de respuesta, exposiciones, predictoras, confusoras y modificacoras del efecto. } \\
\text { Si procede, proporcione los criterios diagnósticos }\end{array}$ \\
\hline Fuentes de datos/medidas & $8^{*}$ & $\begin{array}{l}\text { Para cada variable de interés, proporcione las fuentes de datos y los detalles de los métodos de valoración (medida). } \\
\text { Si hubiera más de un grupo, especifique la comparabilidad de los procesos de medida }\end{array}$ \\
\hline $\begin{array}{l}\text { Sesgos } \\
\text { Tamanio muestral }\end{array}$ & $\begin{array}{l}9 \\
10\end{array}$ & $\begin{array}{l}\text { Especifique todas las medidas adoptadas para afrontar fuentes potenciales de sesgo } \\
\text { Explique cómo se determinó el tamano muestral }\end{array}$ \\
\hline Variables cuantitativas & 11 & Explique cómo se trataron las variables cuantitativas en el análisis. Si procede, explique qué grupos se definieron y por qué \\
\hline Métodos estadisticos & 12 & $\begin{array}{l}\text { (a) Especifique todos los métodos estadisticos, incluidos los empleados para controlar los factores de confusión } \\
\text { (b) Especifique todos los métodos utilizados para analizar subgrupos e interacciones } \\
\text { (c) Explique el tratamiento de los datos ausentes (missing data) } \\
\text { (d) Estudio de cohortes: si procede, explique cómo se afrontan las pérdidas en el seguimiento } \\
\text { Estudios de casos y controles: si procede, explique cómo se aparearon casos y controles } \\
\text { Estudios transversales: si procede, especifique cómo se tiene en cuenta en el análisis la estrategia de muestreo } \\
\text { (e) Describa los análisis de sensibilidad }\end{array}$ \\
\hline
\end{tabular}

\begin{tabular}{|c|c|c|}
\hline \multicolumn{3}{|l|}{ Resultados } \\
\hline Participantes & $13^{*}$ & $\begin{array}{l}\text { (a) Describa el número de participantes en cada fase del estudio; por ejemplo: cifras de los participantes potencialmente } \\
\text { elegibles, los analizados para ser incluidos, los confirmados elegibles, los incluidos en el estudio, los que tuvieron un seguimiento } \\
\text { completo y los analizados } \\
\text { (b) Describa las razones de la pérdida de participantes en cada fase } \\
\text { (c) Considere el uso de un diagrama de flujo }\end{array}$ \\
\hline Datos descriptivos & $14^{*}$ & $\begin{array}{l}\text { (a) Describa las caracteristicas de los participantes en el estudio (p. ej., dernográficas, clinicas, sociales) y la información } \\
\text { sobre las exposiciones y los posibles factores de confusión } \\
\text { (b) Indique el número de participantes con datos ausentes en cada variable de interés } \\
\text { (c) Estudios de cohortes: resuma el periodo de seguimiento (p. ej., promedio y total) }\end{array}$ \\
\hline $\begin{array}{l}\text { Datos de las variables } \\
\text { de resultado }\end{array}$ & $15^{*}$ & $\begin{array}{l}\text { Estudios de cohortes: describa el número de eventos resultado, o bien proporcione medidas resumen a lo largo del tiempo } \\
\text { Estudios de casos y controles: describa el número de participantes en cada categoria de exposición, o bien proporcione medidas } \\
\text { resumen de exposición }\end{array}$ \\
\hline Resultados principales & 16 & $\begin{array}{l}\text { Estudios transversales: describa el número de eventos resultado, o bien proporcione medidas resumen } \\
\text { (a) Proporcione estimaciones no ajustadas y, si procede, ajustadas por factores de confusión, así como su precisión } \\
\text { (p. ej., intervalos de confianza del } 95 \% \text { ). Especifique los factores de confusión por los que se ajusta y las razones para incluirlos } \\
\text { (b) Si categoriza variables continuas, describa los límites de los intervalos } \\
\text { (c) Si fuera pertinente, valore acompanar las estimaciones del riesgo relativo con estimaciones del riesgo absoluto para un } \\
\text { periodo de tiempo relevante }\end{array}$ \\
\hline Otros análisis & 17 & Describa otros análisis efectuados (de subgrupos, interacciones o sensibilidad) \\
\hline \multicolumn{3}{|l|}{ Discusión } \\
\hline Resultados clave & 18 & Resuma los resultados principales de los objetivos del estudio \\
\hline Limitaciones & 19 & $\begin{array}{l}\text { Discuta las limitaciones del estudio, teniendo en cuenta posibles fuentes de sesgo o de imprecisión. Razone tanto sobre } \\
\text { la dirección como sobre la magnitud de cualquier posible sesgo }\end{array}$ \\
\hline Interpretación & 20 & $\begin{array}{l}\text { Proporcione una interpretación global prudente de los resultados considerando objetivos, limitaciones, multiplicidad } \\
\text { de análisis, resultados de estudios similares y otras pruebas empiricas relevantes }\end{array}$ \\
\hline Generabilidad & 21 & Discuta la posibilidad de generalizar los resultados (validez externa) \\
\hline \multicolumn{3}{|l|}{ Otra información } \\
\hline & 22 & $\begin{array}{l}\text { Especifique la financiación y el papel de los patrocinadores del estudio y, si procede, del estudio previo en el que se basa el } \\
\text { presente articulo }\end{array}$ \\
\hline
\end{tabular}

Nota: Se ha publicado un articulo que explica y detalla la elaboración de cada punto de la lista, y se ofrece el contexto metodológico y ejemplos reales de comunicación transparentete-20. La lista de puntos STROBE se debe util izar preferiblemente junto con ese articulo ( $g$ ratuito en las páginas web de las revistas PLOS Medicine [hitp://Wuw.plosmedicine.org/], Annals of Interna/ Medicine [hthtp:/www.annals.org/] y Epidemiology [http://www.epidem.com/]). En la página web de STROBE (http://www.strobe-statement.org) aparecen las diferentes versiones de la lista correspondiente a los estudios de cohortes, a los estudios de casos y controles y a los estudios transversales.

*Proporcione esta información por separado para casos y controles en los estudios con diseño de casos y controles. Si procede, también de los grupos con y sin exposición en los estudios de cohortes y en los transversales. 